\title{
Star formation near an obscured AGN
}

\section{Variations in the initial mass function ${ }^{\star}$}

\begin{abstract}
S. Hocuk and M. Spaans
Kapteyn Astronomical Institute, University of Groningen, PO Box 800, 9700 AV Groningen, The Netherlands

e-mail: [seyit; spaans] aastro.rug.nl

Received 7 June 2011 / Accepted 29 September 2011

ABSTRACT

The conditions that affect the formation of stars in radiatively and mechanically active environments are quite different from the conditions that apply to our local interstellar neighborhood. In these galactic environments, a variety of feedback processes can play a significant role in shaping the initial mass function (IMF). Here, we present a numerical study on the effects of an accreting black hole and the influence of nearby massive stars to a collapsing, $800 M_{\odot}$, molecular cloud at $10 \mathrm{pc}$ distance from the black hole. Our work focusses on the star-forming ISM in the centers of (ultra-)luminous infrared galaxies ((U)LIRGS). We therefore assume that this region is enshrouded by gas and dust and that most of the UV and soft X-ray radiation from the broad line region (BLR) is attenuated along the line of sight to the model cloud. We then parametrize and study radiative feedback effects of hard X-rays emanating from the black hole BLR, increased cosmic ray rates caused by supernovae in starbursts, and strong UV radiation produced by nearby massive stars. We also investigate the importance of shear from the supermassive, $10^{6}-10^{8} M_{\odot}$, black hole as the star-forming cloud orbits around it. A grid of 42 models is created and calculated with the hydrodynamical code FLASH. We find that thermal pressure from X-rays compresses the cloud, which induces a high star-formation rate early on, but reduces the overall star-formation efficiency (SFE) to about 7\% through gas depletion by evaporation. We see that the turn-over mass of the IMF increases up to a factor of 2.3 , $M_{\text {turn }}=1-1.5 M_{\odot}$, for the model with the highest X-ray flux $\left(160 \mathrm{erg} \mathrm{s}^{-1} \mathrm{~cm}^{-2}\right)$, while the high-mass slope of the IMF becomes $\Gamma \gtrsim-1\left(\Gamma_{\text {Salpeter }}=-1.35\right)$. This results in more high-mass stars and a non-Salpeter IMF. Cosmic rays penetrate deeply into the cloud and increase the gas temperature to about $50 \mathrm{~K}$ for rates that are roughly 100 times Galactic and $200 \mathrm{~K}$ for 3000 times Galactic, which leads to a reduced formation efficiency of low-mass stars. While the shape of the mass function is preserved, high cosmic ray rates increase the average mass of stars, thereby shifting the turn-over mass to higher values, i.e., up to several solar masses. Owing to this process, the onset of star formation is also delayed. We find that UV radiation plays only a minor role. Because UV photons cannot penetrate a dense, $n \geqslant 10^{5} \mathrm{~cm}^{-3}$, cloud deep enough, they only affect the late time accretion by heating the medium where the cloud is embedded in. When we increase the black hole mass, for a cloud that is at $10 \mathrm{pc}$ distance, the turbulence caused by shearing effects reduces the SFE slightly. Furthermore, shear weakens the effect of the other parameters on the slope of the IMF as well as the turn-over mass. The run with the most massive black hole, however, causes so much shear that the hydrodynamics is completely dominated by this effect and it severely inhibits star formation. We conclude that the IMF inside active galaxies is different from the one obtained from local environments. We also find that the combined effects of X-rays, cosmic rays, UV, and shear tend to drive toward a less pronounced deviation from a Salpeter IMF.
\end{abstract}

Key words. radiative transfer - methods: numerical - stars: formation - stars: luminosity function, mass function - cosmic rays X-rays: ISM

\section{Introduction}

Star formation in extreme environments can be quite different from the formation of most stars in the Universe. In the inner kpc of galaxies, molecular clouds are exposed to intense radiation from active galactic nuclei (AGN) or starbursts (Maloney et al. 1996; Meijerink \& Spaans 2005; Pérez-Beaupuits et al. 2009; van der Werf et al. 2010). Very close to the AGN, $\lesssim 0.1 \mathrm{pc}$, gas collects into a massive AGN disk over some time, and as the disk becomes unstable, stars are able to form (Paumard et al. 2006; Nayakshin et al. 2007). Slightly farther away from the black hole, 1-100 pc, conditions are somewhat less extreme because the radiation is strongly attenuated by large columns of gas and dust. These environments are typical of obscured AGN, as formed in (U)LIRGS, with obscuring columns of $10^{22}-10^{23.5} \mathrm{~cm}^{-2}$ (Aalto 2005; Pérez-Beaupuits et al. 2007; Loenen et al. 2008). These regions have a strong impact on the initial phases of cloud evolution so that the final mass of stars or

^ Figures 6-17 are available in electronic form at http://www . aanda.org their formation efficiencies might drastically change. However, observing star formation in extreme environments is difficult. Results usually rely on indirect methods and are therefore often debated. The regions near AGN are also generally obscured (Spoon et al. 2007; Hocuk \& Barthel 2010). Predictions based on models and numerical simulations can aid observations to further our understanding of star formation. A good amount of numerical work has been done focussing on mechanical and radiative feedback effects in active galaxies and star-forming regions (Klessen 2001; Klessen et al. 2005a; Bonnell \& Rice 2008; Alexander et al. 2008; Wada 2008; Hobbs \& Nayakshin 2009; Wada et al. 2009; Krumholz et al. 2010; Bate 2010; Hocuk \& Spaans 2010b,a; Pérez-Beaupuits et al. 2011; Clark et al. 2011; Latif et al. 2011; Aykutalp \& Spaans 2011; Alig et al. 2011). Supported by numerical simulations, it is often thought that in these active regions, the IMF should be different from the proposed universal mass function because of environmental conditions and feedback effects (Klessen et al. 2007; Nayakshin et al. 2007; Hsu et al. 2010; Krumholz et al. 2010; Girichidis et al. 2011). 
The IMF is of fundamental importance for many areas of astrophysics. It is observed to behave like a power-law with a high-mass end that is well-defined. The IMF is described as

$\frac{\mathrm{d} N}{\mathrm{~d} M} \propto M^{-\alpha} \Longrightarrow \frac{\mathrm{d} \log N}{\mathrm{~d} \log M}=-\alpha+1=\Gamma$,

with $N$ the number of stars in a range of mass $\mathrm{d} M, \alpha$ the powerlaw index, and $\Gamma$ the slope above the characteristic mass of $\sim 0.3-0.5 M_{\odot}$. First proposed by Salpeter (1955), a plethora of observations has led astronomers to believe that the shape might be universal in nature. Other astronomers have refined the shape of the distribution by especially improving the low-mass end of the IMF (Miller \& Scalo 1979; Kroupa 2001; Chabrier 2003). Until observations of extragalactic origin started to show variations in the IMF, most of the studies that claimed universality were coming from observations from our local neighborhood. Hints for deviation came from measurements of abundance patterns in extragalactic bulges (Ballero et al. 2007, 2008), enhancement of far infra-red luminosities in interacting galaxy systems (Brassington et al. 2007), mass-to-light ratios of ultra-compact dwarf galaxies (Dabringhausen et al. 2009), and many others (Baugh et al. 2005; Parra et al. 2007; Davé 2008; Wilkins et al. 2008; van Dokkum 2008; Elmegreen 2009). van Dokkum \& Conroy $(2010,2011)$ found additional evidence for variations in the IMF at the low-mass end from $\mathrm{NaI}$ and $\mathrm{FeH}$ band spectra in luminous elliptical galaxies. Still, strong evidence has yet to emerge.

Although the IMF may seem, theoretically, to be universal over a relatively wide range of environmental conditions (Clark et al. 2009; Bastian et al. 2010; Krumholz 2011), perhaps even insensitive to small changes in metallicity (Myers et al. 2011) and the Jeans mass (Elmegreen et al. 2008), there are conditions that are far more extreme than those discussed in these papers, such as radiation-dominated regions (Meijerink et al. 2005; Poelman \& Spaans 2006; van der Werf et al. 2010; Meijerink et al. 2011) and cosmic ray dominated regions (CRDRs, Papadopoulos et al. 2011). Besides the importance of the thermodynamics for the Jeans mass, $M_{\mathrm{J}}$, and thus the IMF, where $M_{\mathrm{J}}$ is proportional to $\rho^{-1 / 2} T^{3 / 2}$, the change in the equation of state is also essential. Assuming ideal gas conditions with a polytropic equation of state, $P \propto \rho^{\gamma}$, where $\gamma=1+\operatorname{dlog}(\mathrm{T}) / \operatorname{dlog}(\rho)$, the softness of $\gamma$ plays a major role at a very early stage in the fragmentation properties and the mass scale of unstable clouds (Spaans \& Silk 2000; Li et al. 2003; Klessen et al. 2005b; Jappsen et al. 2005).

The nuclei of active galaxies such as Arp 220, Markarian 231, and even our Galactic center show signs of unusual star formation (Figer 2005a,b; Paumard et al. 2006; Klessen et al. 2007; Espinoza et al. 2009; Elmegreen 2009; Bartko et al. 2010; Matsushita et al. 2009; Meijerink et al. 2011; Martín et al. 2011). Indeed, even in the inner parsec of our Galaxy, i.e., Sgr A* and in M 31, young stars are found at distances on the order of 0.03-0.3 pc (Genzel et al. 2003; Paumard et al. 2006; Levin 2007). All the aforementioned places harbor a massive black hole. One can imagine that the conditions close to the black hole can indeed become quite extreme. Aside from strong gravity, accreting material onto a black hole will produce strong X-ray radiation (1-100 KeV). The dynamics of molecular clouds will be significantly affected by the irradiation of X-rays in X-ray dominated regions (XDRs, Lepp \& Dalgarno 1996; Maloney et al. 1996). On the other hand, in starbursts, where star-formation rates can be a few hundred to a thousand solar masses per year (Smail et al. 1997; Hughes et al. 1998), UV radiation $(6-13.6 \mathrm{eV})$ from $\mathrm{O}$ and $\mathrm{B}$ stars can be a significant presence and dominate the radiation field in these so-called photon-dominated regions (PDRs, Hollenbach \& Tielens 1999). However, where the gas is shielded from UV radiation, cosmic rays, created in supernova remnants or from winds in OB associations (Binns et al. 2008) with energy densities of up to a few thousand times our galaxy, will dictate the (minimum) temperature of the system (Papadopoulos 2010; Papadopoulos et al. 2011; Meijerink et al. 2011). All these environments have extremely different star-forming conditions, but will the stars that form out of them be much different?

In radiation-dominated regions, the chemistry and thermal balance are determined by the radiation field (Schleicher et al. 2010). X-rays in AGN are usually the dominant source for the excitation and chemistry of the inner disk out to a radius of $\sim 160 \mathrm{pc}$, while UV radiation dominates the thermal balance in extreme starbursts and is generally important a bit farther away from the accreting black hole (van der Werf et al. 2010). But there is compelling evidence that there is a strong link between AGN and starbursts (Scoville 2004). The question remains, how strongly star formation is affected by these extreme environments.

In an earlier numerical study, we showed that when the X-ray flux is as high as $160 \mathrm{erg} \mathrm{s}^{-1} \mathrm{~cm}^{-2}$, the stellar IMF of an 800 solar mass molecular cloud becomes top-heavy (Hocuk \& Spaans 2010a). This was the case where a molecular cloud orbiting at $10 \mathrm{pc}$ from a $10^{7} M_{\odot}$ black hole under the impact of X-rays was compared to a cloud with the same conditions but in an $\mathrm{X}$-ray-free environment. This numerical study showed that under extreme conditions the evolution of a molecular cloud and its stellar mass function will change, but it did not give insight into the quantitative details. Here, we present a parameter study on the influence of external radiation (X-rays, cosmic rays, and UV) and black hole shear on the IMF and the SFE. In Sect. 2 we introduce the numerical code FLASH and describe our additions to it. In Sect. 3 we define the cloud models for all ambient conditions considered in the parameter study. We then present in Sect. 4 our results on the effects of each condition for the evolution of the model clouds and show their phase diagrams, SFEs, and initial mass functions. Finally, in Sect. 5, we discuss the differences and similarities of these results in detail and present our conclusions.

\section{Computational method}

\subsection{The numerical code}

The calculations in this work were made using the hydrodynamical code FLASH 3 (Dubey et al. 2009). For this study, we used the directionally split piecewise-parabolic method (PPM), which is described in detail in Colella \& Woodward (1984). FLASH is well-suited to handle these types of calculations because it is an adaptive mesh code and one that handles contact discontinuities very well. FLASH is provided with many and extensively tested modules that encompass a broad range of physics. Our simulation code is equipped with thermodynamics, hydrodynamics, (self-)gravity, multi-species, particles, and shocks, all from the standard modules of FLASH, as well as sink particles, radiative transfer, multi-scale turbulence, and refinement criteria (based on Jeans length and particles) that were added by us. The nonstandard additions are explained in more detail in the following sections and in Hocuk \& Spaans (2010a). 


\subsection{Refinement criteria}

When one conducts a parameter study and has to perform many numerical simulations, saving time becomes crucial. To achieve this without loss of quality, we made use of the adaptiveness of the FLASH code and wrote two independent refinement criteria that served our purpose. The simpler one of the two is based on sink particles. Because every sink particle accretes matter, and this matter can only be followed to within the sink particle's accretion radius, it is best to have the highest possible resolution here to resolve the affected volume properly. Particles can only be created in regions that have the highest grid resolution, however, they can move through the grid to unrefined regions. To this end, we simply say that wherever a sink particle is located, the grid must be refined to its maximum.

The second criterion is based on the Jeans length. To avoid numerical effects such as artificial fragmentation (Truelove et al. 1997 ) it is necessary to resolve the Jeans length, $\lambda_{\mathrm{J}}$, in the simulation by at least 4 cells, $\lambda_{\mathrm{J}} \geq 4 \Delta x$. Here, $\Delta x$ is the size of the grid cell, which depends on the resolution. In our experience, however, it is also likely that fragmentation can be artificially inhibited at even higher resolutions, up to twice the Truelove criterion, that is, 8 cells. In the presence of magnetic fields, even higher resolution constraints are found by Federrath et al. (2011). However, we do not consider magnetic field effects in this study. Therefore, we have chosen to resolve the Jeans length in our simulations by at least 10 grid cells, $\ell_{\text {res,gas }}=10$. This refinement criterion can be rewritten in the form of a density threshold and refines the grid if the following condition is met:

$\rho \geq \frac{M_{\mathrm{J}}}{\frac{4}{3} \pi\left(\ell_{\mathrm{res}} \triangle x\right)^{3}}$,

with the Jeans mass, $M_{\mathrm{J}}$, defined as

$M_{\mathrm{J}}=\frac{4}{3} \pi \lambda_{\mathrm{J}}^{3} \rho=\left(\frac{\pi c_{\mathrm{s}}^{2}}{G}\right)^{\frac{3}{2}} \rho^{-\frac{1}{2}} \simeq \frac{90}{\mu^{2}} T^{\frac{3}{2}} n^{-\frac{1}{2}}\left(M_{\odot}\right)$,

as taken from Frieswijk et al. (2007). Using this, Eq. (2) can be further reduced to

$\rho \geq 1.455 \times 10^{15} \frac{T}{\mu\left(\ell_{\mathrm{res}} \Delta x\right)^{2}}$,

where $\mu$ is the mean molecular weight, $G$ is the gravitational constant, and $T$ is the gas temperature.

Because we have a body in orbit, the Jeans refinement criterion will automatically follow the cloud in motion and increase the resolution of grid cells whenever required. The border resolution between the refinement levels is set up in such a way that there is no sharp transition between the minimum and the maximum refinement. To be efficient, we also de-refined the regions where the Jeans length was stretched beyond 25 times the grid resolution. One can imagine this as a region where the cloud has just passed through.

\subsection{Multi-scale turbulence}

Turbulence is an important aspect of star formation. The interstellar matter from which stars form is never fully homogeneous or kinematically quiescent. Typical velocity dispersions are found to be on the order of $1 \mathrm{~km} \mathrm{~s}^{-1}$ in most of the regions in our galaxy (Larson 1981; Falgarone et al. 2001; Caselli et al. 2002) and scale according to a power-law. It should be no surprise that gaseous clouds within active regions are more turbulent. Typical $F W H M$ values are on the order of $5 \mathrm{~km} \mathrm{~s}^{-1}$ (Pérez-Beaupuits et al. 2009; Ostriker \& Shetty 2011).

Numerical simulations have shown that turbulent strength, scaling, or type (compressible or solenoidal) can indeed be quite important and affect the results significantly (Federrath et al. 2009; Girichidis et al. 2011). It is therefore important to incorporate turbulence into the numerical code in a proper way. In all our simulations, we implemented the turbulence using a Larson power-law, with a power spectrum $P(k) \propto k^{-4}$ and thus $\Delta v \propto \ell^{1 / 2}$, where $k \propto \ell^{-1}$ is the scale length (Larson 1981; Myers \& Gammie 1999; Heyer \& Brunt 2004). This is the predicted and observed behavior for compressible fluids. Because the grids in hydrodynamical simulations are discretized, and in block-structured grids the cell sizes usually increase with a factor of two for each resolution increment, the super-posed velocity decreases with the square-root of two for each higher level. In our case, the largest scale that we apply the multi-scale turbulence on is that of the cloud radius. Otherwise, the cloud as a whole would obtain a random motion. The smallest scale on which the turbulence is injected is determined by the maximum resolution at runtime, which is $\Delta x=1.76 \times 10^{16} \mathrm{~cm}$ in our runs.

\subsection{The radiative transfer method}

Radiative transfer for PDRs and XDRs is handled through a radiation-dominated region code written by Meijerink \& Spaans (2005), with additional details in Meijerink et al. (2007) and Spaans \& Meijerink (2008). Pre-computed tables for gas temperature and chemical abundances were obtained from this code given an input of number density $\left[\mathrm{cm}^{-3}\right]$, radiation flux [erg s $\mathrm{cm}^{-1}$ ] for X-rays and UV, column density $\left[\mathrm{cm}^{-2}\right]$, and metallicity. We chose solar abundances for all simulations. For the regions dominated by radiation, the code calculates all heating processes (photo-ionization, yielding non-thermal electrons, FUV pumping followed by collisional de-excitation), cooling processes (atomic fine-structure and semi-forbidden lines), and molecular transitions ( $\mathrm{CO}, \mathrm{H}_{2}, \mathrm{H}_{2} \mathrm{O}, \mathrm{OH}$, and $\left.\mathrm{CH}\right)$. Cosmic rays, dust-gas coupling, and secondary effects from X-rays such as internal UV are treated as well. These tables were incorporated into FLASH. For the cloud models in the absence of radiation, we used isothermal conditions with an equation of state of the form $P \propto \rho$ (Hocuk \& Spaans 2010a,b).

We used a ray-tracing method to find the column densities during the simulation. At each time-step, the algorithm searches the grid and sums up the densities of each cell lying along the line of sight from the radiation source to find the total column. The cells were selected by determining whether the two angles of the cell edges with respect to the radiation source accommodate the angle of the target cell. Each cell was weighted with the length of the ray that passes through it. Once the column density was found, together with the density and the flux, the corresponding temperature was taken from the tables and used to update the variables in the simulation. See Pérez-Beaupuits et al. (2011) for additional details. To gain speed, the algorithm makes use of the block-structured mesh of FLASH by first searching the blocks that lie within the line of sight of the source, thereby only looking into the cells of those blocks. This makes the ray-tracing in effect about 500 times faster. This gain is welcome, because by far the most computational time is normally spent on finding the column densities. Additional speed was gained by not applying the ray-tracing algorithm to the whole grid at every timestep. We prioritized the regions that have densities above $100 \mathrm{~cm}^{-3}$ and updated them regularly, but the lower density regions were only updated occasionally. These regions are not very important 
because stars cannot form here. Besides, the temperatures at low densities do not decrease quickly because the cooling time scales as $t_{\text {cool }} \propto 1 / n$ (optically thin and sub-thermal), and regular updates are not necessary.

The X-ray flux is an $E^{-0.9}$ power-law between 1 and $100 \mathrm{keV}$. $\mathrm{X}$-ray scattering is not very important, but is nonetheless treated in the XDR-code. Inverse-Compton heating was not included, because our focus lies on the molecular gas $(<1000 \mathrm{~K})$, which is mostly heated by ionization of $\mathrm{H}_{\text {and }} \mathrm{H}_{2}$, and by $\mathrm{H}_{3}^{+}$recombination (Meijerink \& Spaans 2005). A uniform background of cosmic rays prevented the temperature from dropping below $10 \mathrm{~K}$. For this, a minimum cosmic ray ionization rate typical for the Milky Way, $\zeta_{\mathrm{CR}, \mathrm{Gal}}=5 \times 10^{-17} \mathrm{~s}^{-1}$, was assumed $($ Spaans $\&$ Silk 2000). The UV flux enjoys energies between 6 and $13.6 \mathrm{eV}$ and follows the Habing spectrum (Habing 1968).

\subsection{Sink particles}

Sink particles are necessary if one wants to obtain a high dynamic range in density. These particles represent compact (proto-stellar) objects that are indivisible but can gain mass by accreting or merging. It is computationally very expensive to keep increasing the grid resolution to follow a molecular cloud collapse up to proto-stellar densities. We created a sink particle algorithm for this purpose. There are several criteria that we checked to establish irreversible collapse. When this stage was reached, one could stop following the collapse and could make a transition to sink particles. Following Bate et al. (1995) and Federrath et al. (2010), with only small differences, we determine the point-of-no-return of a grid cell within a volume that is defined by an accretion radius $r_{\text {acc }}$ as

1. The grid cell is about to violate the Jeans criterion, Eq. (4).

2. The grid cell and its neighbors are at the highest level of refinement.

3. The grid cell has the deepest gravitational potential of all cells within the volume.

4. The divergence on each axis of the grid cell is negative, that is, $\mathrm{d} v_{i} / \mathrm{d} i<0$, with $i=\{x, y, z\}$, such that $\nabla \cdot \mathbf{v}<0$.

5. The volume within the accretion radius is gravitationally bound, $E_{\text {grav }}+E_{\text {th }}+E_{\text {kin }}<0$.

6. The volume within the accretion radius is Jeans-unstable, $E_{\text {grav }}+2 E_{\text {th }}<0$ and thus $M>M_{\mathrm{J}}$.

7. There are no other sink particles occupying the same cell.

At the moment when a sink particle is created, material from within an accretion radius is taken away from the cells and put into the particle. We made sure that the total mass and momentum were conserved. The accretion radius is ideally smaller than the sonic radius, $C_{\mathrm{s}} \mathrm{dt}$, but we also aimed to resolve this region well. On the other hand, we aimed to keep the radius small, because we changed the physics within this radius. In practice we found that two cells were a reasonable compromise between these competing demands, see also Krumholz et al. (2004) and Federrath et al. (2010). Our accretion radius is $r_{\mathrm{acc}}=3.5 \times 10^{16} \mathrm{~cm}$ and reproduces the observed Chabrier IMF for runs M 01 and M 04, see Sect. 4.

The mass that a sink particle obtains at creation is determined by a density threshold that is set by the maximum resolution. In this case, we followed the same resolution criterion as previously mentioned (Truelove et al. 1997), but let the code run as long as possible on gas dynamics before we made the transition to sink particles. Using Eqs. (2) and (4), and taking $\ell_{\text {res, sink }}=6$, we determined how much material within $r_{\text {acc }}$ from the target cell was in excess of the threshold density and added this to the mass of the particle. Sink particles continue to accrete matter after they are created. Accretion onto particles is handled by a Bondi-Hoyle type of accretion (Bondi \& Hoyle 1944; Bondi 1952; Ruffert \& Arnett 1994; Krumholz et al. 2004). This kind of accretion applies to a homogeneous flow of matter. Accretion increases with protostellar mass $M$, but drops with decreasing ambient density $\rho_{\infty}$ and higher Mach numbers $\mathcal{M}$. The accretion rate is given by

$\dot{M}=4 \pi \rho_{\infty} G^{2} M^{2} \sqrt{\frac{\lambda^{2} c_{\infty}^{2}+v_{\infty}^{2}}{\left(c_{\infty}^{2}+v_{\infty}^{2}\right)^{4}}}=\frac{4 \pi \rho_{\infty} G^{2} M^{2}}{c_{\infty}^{3}} \sqrt{\frac{\lambda^{2}+M^{2}}{\left(1+M^{2}\right)^{4}}}$,

where $c_{\infty}$ and $v_{\infty}$ are the sound speed and the velocity of the gas far from the sink particle. Because there is no obvious choice for $c_{\infty}$ and $v_{\infty}$ in an inhomogeneous environment, we took the average value of the cells inside $r_{\text {acc }}$ as an alternative. $\lambda$ is a nondimensional parameter that depends on the equation of state of the gas and is on the order of unity. Throughout this work, we adopted the value for an isothermal gas $\lambda=\exp (3 / 2) / 4 \simeq 1.12$ (Bondi 1952).

Using the effective radius as first described by Bondi (1952)

$r_{\mathrm{BH}}=\frac{G M}{c_{\infty}^{2}+v_{\infty}^{2}}$,

Eq. (5) can be additionally reduced to

$\dot{M}=4 \pi \rho_{\infty} r_{\mathrm{BH}}^{2} c_{\infty} \sqrt{\lambda^{2}+\mathcal{M}^{2}}$,

where $\rho_{\infty}$ is defined as

$\rho_{\infty}=\frac{\bar{\rho}}{\alpha}$.

Here, $\bar{\rho}$ is the mean density within the accretion radius and $\alpha$ is the density profile that depends on the cell size and the Bondiradius. For the density profile, we used an exponential of the form $\alpha=\exp \left(r_{\mathrm{BH}} / 1.2 \triangle x\right)$. We found this expression to behave well in the regime $\Delta x \gtrsim r_{\mathrm{BH}}$, where we usually are in, and it is equal to unity when $\Delta x \gg r_{\mathrm{BH}}$. The factor of 1.2 was adopted from Krumholz et al. (2004) and should give good results in the range where $\Delta x \sim r_{\mathrm{BH}}$.

Stars are often found in binaries, however, we cannot resolve binaries in our numerical code because our resolution is on the order of $2000 \mathrm{AU}$. Instead, we allowed them to merge. Binary formation or mergers should occur more frequently as the system virializes. However, these mergers do not affect our conclusions, see Sect. 5. Sink particles are eligible to merge when they are within each other's gravitational pull. We let an algorithm check for three conditions between every two particles, and when they passed these, we allowed them to merge. This happens when

1. The velocity difference between two particles, $i$ and $j$, is less than the escape velocity between them, $\Delta v_{i j}<\sqrt{2 G M_{i} / \Delta r_{i j}}$, with $M_{i}>M_{j}$.

2. The merging time is shorter than the hydrodynamical timestep, $\pi \Delta r_{i j}^{3 / 2} / \sqrt{8 G\left(M_{i}+M_{j}\right)}<\mathrm{d} t_{\text {hydro }}$.

3. The forces of the other bodies, $F_{\text {tot }}$, are no longer significant, i.e., $F_{\text {tot }}<0.05 \times F_{i j}$, where $F_{\mathrm{ij}}=G M_{i} M_{j} / \Delta r_{i j}^{2}$.

\section{Models and initial conditions}

\subsection{A grid of models}

We computed a grid which consists of 42 models that covers a range in the four parameters that we investigate. As stated before, the parameters that we varied are the X-ray flux, the cosmic 
Table 1. Parameter details for each model.

\begin{tabular}{cccccccccc}
\hline \hline Model & $\begin{array}{c}F_{\mathrm{X}} \\
{\left[\mathrm{erg} \mathrm{s}^{-1} \mathrm{~cm}^{-2}\right]}\end{array}$ & $\begin{array}{c}M_{\mathrm{bh}} \\
{\left[M_{\odot}\right]}\end{array}$ & $\begin{array}{c}\zeta_{\mathrm{CR}} \\
{[\times \mathrm{Galactic}]}\end{array}$ & $\begin{array}{c}F_{\mathrm{UV}} \\
{\left[\mathrm{G}_{0}\right]}\end{array}$ & Model & $\begin{array}{c}F_{\mathrm{X}} \\
{\left[\mathrm{erg} \mathrm{s}^{-1} \mathrm{~cm}^{-2}\right]}\end{array}$ & $\begin{array}{c}M_{\mathrm{bh}} \\
{\left[M_{\odot}\right]}\end{array}$ & $\begin{array}{c}\zeta_{\mathrm{CR}} \\
{[\times \mathrm{Galactic}]}\end{array}$ & $\begin{array}{c}F_{\mathrm{UV}} \\
{\left[\mathrm{G}_{0}\right]}\end{array}$ \\
\hline M 01 & 0 & $10^{7}$ & 1 & 0 & M 22 & 160 & $10^{6}$ & 1 & 0 \\
M 02 & 0 & $10^{7}$ & 100 & 0 & M 23 & 160 & $10^{6}$ & 100 & 0 \\
M 03 & 0 & $10^{7}$ & 3000 & 0 & M 24 & 160 & $10^{6}$ & 3000 & 0 \\
M 04 & 0 & $10^{6}$ & 1 & 0 & M 25 & 0 & $10^{8}$ & 1 & 0 \\
M 05 & 0 & $10^{6}$ & 100 & 0 & M 26 & 0 & $10^{8}$ & 100 & 0 \\
M 06 & 0 & $10^{6}$ & 3000 & 0 & M 27 & 0 & $10^{8}$ & 3000 & 0 \\
M 07 & 5.1 & $10^{7}$ & 1 & 0 & M 28 & 160 & $10^{8}$ & 1 & 0 \\
M 08 & 5.1 & $10^{7}$ & 100 & 0 & M 29 & 160 & $10^{8}$ & 100 & 0 \\
M 09 & 5.1 & $10^{7}$ & 3000 & 0 & M 30 & 160 & $10^{8}$ & 3000 & 0 \\
M 10 & 5.1 & $10^{6}$ & 1 & 0 & M 31 & 0 & $10^{7}$ & 1 & $10^{2.5}$ \\
M 11 & 5.1 & $10^{6}$ & 100 & 0 & M 32 & 0 & $10^{7}$ & 100 & $10^{2.5}$ \\
M 12 & 5.1 & $10^{6}$ & 3000 & 0 & M 33 & 0 & $10^{7}$ & 3000 & $10^{2.5}$ \\
M 13 & 28 & $10^{7}$ & 1 & 0 & M 34 & 5.1 & $10^{7}$ & 1 & $10^{2.5}$ \\
M 14 & 28 & $10^{7}$ & 100 & 0 & M 35 & 5.1 & $10^{7}$ & 100 & $10^{2.5}$ \\
M 15 & 28 & $10^{7}$ & 3000 & 0 & M 36 & 5.1 & $10^{7}$ & 3000 & $10^{2.5}$ \\
M 16 & 28 & $10^{6}$ & 1 & 0 & M 37 & 28 & $10^{7}$ & 1 & $10^{2.5}$ \\
M 17 & 28 & $10^{6}$ & 100 & 0 & M 38 & 28 & $10^{7}$ & 100 & $10^{2.5}$ \\
M 18 & 28 & $10^{6}$ & 3000 & 0 & M 39 & 28 & $10^{7}$ & 3000 & $10^{2.5}$ \\
M 19 & 160 & $10^{7}$ & 1 & 0 & M 40 & 160 & $10^{7}$ & 1 & $10^{2.5}$ \\
M 20 & 160 & $10^{7}$ & 100 & 0 & M 41 & 160 & $10^{7}$ & 100 & $10^{2.5}$ \\
M 21 & 160 & $10^{7}$ & 3000 & 0 & M 42 & 160 & $10^{7}$ & 3000 & $10^{2.5}$ \\
\hline
\end{tabular}

ray rate, black hole mass, and the UV flux. A $10^{7} M_{\odot}$ black hole accreting at $10 \%$ Eddington and at a distance of $10 \mathrm{pc}$, would radiate with a total flux of $F_{\text {Edd }}=10^{4}\left(M_{\mathrm{bh}} / 10^{7} M_{\odot}\right) \mathrm{erg} \mathrm{s}^{-1} \mathrm{~cm}^{-2}$. Because we are interested in (U)LIRGS, we assumed that there is a large absorbing column with $\tau_{1 \mathrm{keV}} \sim 5$ and $\tau_{\mathrm{UV}} \gtrsim 50$ between our single star-forming cloud and the black hole BLR. Varying the black hole mass $M_{\mathrm{bh}}$ or its distance from the model cloud $d_{\mathrm{bh}}$, gives the same insight into the effects of gravitational shear, where the largest velocity difference $\Delta v$ across the cloud depends on $M_{\mathrm{bh}} / d_{\mathrm{bh}}^{3}$ for a given model cloud size much smaller than $d_{\text {bh }}$. We chose to fix the cloud distance to the black hole and vary the shear using the black hole mass.

We studied four X-ray fluxes by varying the Eddington rates, these are: $0,5.1,28,160 \mathrm{erg} \mathrm{s}^{-1} \mathrm{~cm}^{-2}$, with roughly a factor of 5 between them. X-ray fluxes much higher than $100 \mathrm{erg} \mathrm{s}^{-1} \mathrm{~cm}^{-2}$ would completely ionize any molecular cloud of $\lesssim 10^{6} \mathrm{~cm}^{-3}$ and inhibit star formation. We considered three black hole masses: $10^{6}, 10^{7}$, and $10^{8} M_{\odot}$, which, for a fixed distance of $d_{\mathrm{bh}}=10 \mathrm{pc}$, represent strong shear, medium shear, and negligible shear, respectively. Furthermore, we investigated three different cosmic ray rates: 1,100 , and 3000 times Galactic $\left(\zeta_{\mathrm{CR}, \mathrm{Gal}}=5 \times 10^{-17} \mathrm{~s}^{-1}\right.$, Spaans \& Silk 2000). Finally, we used two UV fluxes in our simulations for which we considered 0 and $10^{2.5} \mathrm{G}_{0}$, with $G_{0}=$ $1.6 \times 10^{-3} \mathrm{erg} \mathrm{s}^{-1} \mathrm{~cm}^{-2}$ (Habing 1968). For each parameter that we changed, we kept all other conditions fixed. Almost all possible combinations between these parameters, with the exception of UV, were modeled in this study. Our fiducial model M 01 has similar conditions to those of the Milky Way, albeit with added gravitational shear. In this respect, model M 04, which has negligible shear, is more closely related to the conditions of the solar neighborhood. Table 1 shows the parameter details of all models.

\subsection{The initial conditions}

We created a simulation box of size $24^{3} \mathrm{pc}^{3}$ with outflow boundaries in each direction and isolated in terms of gravity. In this, we put a typical molecular cloud, or clump as some prefer, with a uniform number density of $10^{5} \mathrm{~cm}^{-3}$ and a size of $r_{\text {cloud }}=0.33 \mathrm{pc}$ in spherical radius. With a mean molecular weight of $\mu=2.3$, the total mass of the cloud amounted to 800 solar masses. The rest of the medium was filled with gas that had a uniform density of $10 \mathrm{~cm}^{-3}$. The total gas mass of the simulation box was 8000 solar masses. At the center of the box we put a point particle with mass $M_{\mathrm{bh}}$ that represented the black hole, where $M_{\mathrm{bh}}=\left[10^{6}, 10^{7}, 10^{8}\right] M_{\odot}$. The temperature of the gas was initialized as $10 \mathrm{~K}$, but depending on the model we exposed the cloud immediately after the simulation starts to external radiation. Because these radiative processes are fast with respect to the hydrodynamics of the simulation, the temperature changed quickly, within $10^{8} \mathrm{~s}$ (= one timestep), after initialization. The radiation source was either an X-ray emitter (accreting black hole), a uniform background of cosmic rays (mainly supernova remnants), and/or an isotropic UV radiation field (nearby massive stars).

An initially random, divergence-free turbulent velocity field was applied to the molecular cloud with a characteristic FWHM of $5 \mathrm{~km} \mathrm{~s}^{-1}$ that agrees well with clouds observed in active regions (Pérez-Beaupuits et al. 2009). For the isothermal runs, the sound speed of the cloud was $c_{\mathrm{s}}=0.19 \mathrm{~km} \mathrm{~s}^{-1}$ (for $T=10 \mathrm{~K}$ ) and could go up to $\sim 5 \mathrm{~km} \mathrm{~s}^{-1}$ when radiation impinged on the cloud. There were supersonic flows with Mach numbers of up to 25 . We applied the turbulence over all scales with a power spectrum of $P(k) \propto k^{-4}$, as mentioned above, following the empirical laws for compressible fluids (Larson 1981; Myers \& Gammie 1999; Heyer \& Brunt 2004). The turbulence in this work is not driven. Still, it can remain strong throughout a simulation because of gravitational instabilities or shear induced by the black hole, and does so for the larger $M_{\mathrm{bh}}$ runs.

The simulations started with a cloud at $10 \mathrm{pc}$ distance from the black hole which was in a stable Keplerian orbit. The orbital time was on the order of $10^{6} \times\left(10^{7} M_{\odot} / M_{\mathrm{bh}}\right)^{1 / 2} \mathrm{yr}$, which is longer than the cloud free-fall time in any of the models. Shear caused by the black hole gravity, created a maximum velocity difference of about $\Delta v_{\text {shear }}=2.22 \times\left(M_{\mathrm{bh}} / 10^{7} M_{\odot}\right)^{1 / 2} \mathrm{~km} \mathrm{~s}^{-1}$ 
across the cloud. This follows from

$\Delta v_{\text {shear }}=\sqrt{\frac{G M_{\mathrm{bh}} r_{\text {cloud }}^{2}}{d_{\mathrm{bh}}^{3}}}$,

where $r_{\text {cloud }} \ll d_{\text {bh }}$. The shearing time, $t_{\text {shear }}=2 r_{\text {cloud }} / \Delta \mathrm{v}_{\text {shear }}$, is about $1-10$ times longer than the cloud free-fall time and gravitationally bound (roughly) spherical clouds are thus likely to exist at densities of $\sim 10^{5} \mathrm{~cm}^{-3}$.

The maximum grid resolution that we allowed for in any simulation is $4096^{3}$ cells. For a box of size $24 \mathrm{pc}$, the maximum spatial resolution became $\Delta x=1.76 \times 10^{16} \mathrm{~cm}$. All simulations were evolved up to three free-fall times, where one free-fall time, given the initial conditions, was $t_{\mathrm{ff}}=10^{5}$ years. This was taken as the basic time unit throughout this work.

\section{Results}

We divided the results into four sections. In each section we individually evaluated the effects of X-rays, cosmic rays, UV, and shear, while keeping every other parameter fixed. In Figs. 6 to 17 , we first show the results of all parameter variations at once because this gives a better overview. Figures 6 to 9 display the temperature-density phase diagrams, Figs. 10 to 13 the SFEs, and Figs. 14 to 17 the IMFs.

For each phase diagram in Figs. 6 to 9 we plotted the number density versus the temperature for one moment in time, which is at $1 t_{\mathrm{ff}}$. The diagram was subsequently gridded into $75^{2}$ cells and the weighted masses of all points within each cell was summed up and depicted in color. Note that the isothermal conditions always yield a flat profile.

The SFEs in Figs. 10 to 13 are displayed by plotting the ratio of the total sink particle mass over the total initial gas mass $\left(8000 M_{\odot}\right)$, SFE $=M_{\text {sink }} / M_{\text {total }}$, against time. The efficiencies were plotted as they are, i.e., no fitting is involved. Between 1 and $2 t_{\mathrm{ff}}$ the SFEs generally flatten out. This is caused by the depletion of high density gas. At this stage, star formation is almost completely halted and accretion is reduced to a minimum.

Each IMF plot in Figs. 14 to 17 was constructed by taking all sink-particles of 61 different cloud evolution times of one model and by normalizing them. For this, we took the time frames between $1 t_{\mathrm{ff}}$ and $3 t_{\mathrm{ff}}$, with a time resolution of $1 / 30 t_{\mathrm{ff}}$. The sink particle masses were then plotted as a logarithmically binned histogram with a fixed number of 16 bins of stellar mass versus number. We compared these IMFs with those corresponding to the time frames between $1 t_{\mathrm{ff}}$ and $2 t_{\mathrm{ff}}$, and found that there was little difference between them. There are four colored lines plotted in each of the sub-figures. The green line represents the Salpeter IMF with a slope of $\Gamma_{\mathrm{Sal}}=-1.35$ and is shown for comparison purposes only. The blue line displays the Chabrier IMF as fitted to our fiducial case, model $\mathrm{M} 01$, at $t=t_{\mathrm{ff}}$ (this is a single snapshot in time). Model M 01 is an isothermal simulation with $M_{\mathrm{bh}}=10^{7} M_{\odot}, F_{\mathrm{X}}=0, F_{\mathrm{UV}}=0$, and $\zeta_{\mathrm{CR}}=1 \times$ Galactic. See also Hocuk \& Spaans (2010a). This curve is a lognormal function up to $1 M_{\odot}$ with a power-law tail of $\Gamma_{\mathrm{Cha}}=-1.30$ (Chabrier 2003). The red solid line gives the best (least squares) lognormal fit, whereas the purple triple-dotted line shows the best (least squares) power-law fit above the turn-over mass. The turn-over mass is not fixed and can change for each model. We determined the turn-over mass by finding the position with the lowest absolute derivative (slope) $|\mathrm{d} \log N / \mathrm{d} \log M| \rightarrow 0$ from the lognormal fit.

\subsection{Effects of X-rays}

$\mathrm{X}$-rays heat up the lower density unshielded gas and can do so up to $6000 \mathrm{~K}$ at the highest flux. Hard X-rays ( $>1 \mathrm{keV}$ ) have a high penetration factor. They can pierce through columns of gas of up to $N \simeq 10^{24} \mathrm{~cm}^{-2}$ (Meijerink \& Spaans 2005). Because the X-ray source is in the center of the simulation box, material that lies behind high-density gas, with column densities beyond $10^{23} \mathrm{~cm}^{-2}$, can be strongly shielded from the radiation. This occured in our simulations. Our initialized model cloud had a maximum column of $N=2.0 \times 10^{23} \mathrm{~cm}^{-2}\left(\rho=10^{5} \mathrm{~cm}^{-3}\right.$ and $\left.2 r_{\text {cloud }}=2.0 \times 10^{18} \mathrm{~cm}\right)$. Some of the phase diagrams show a secondary line at low densities that exhibits a sharp drop to low temperatures because of the cold shielded gas. In each of the Figs. 6 to 17 the X-rays increase from top to bottom.

From the phase diagrams, it is immediately clear that the gas temperatures decrease with increasing density and that the temperatures are higher for the higher X-ray fluxes at any point in density. The latter is a direct consequence of the efficient Coulomb heating by X-rays. X-rays do not only heat the system, but also allow new paths for cooling to proceed along. X-rays increase the ionization fraction of the species and new molecules are formed. This results in a higher cooling efficiency with increasing density, hence, the decreasing temperature trend. We can also see a spread in the temperature as a direct effect from the different column densities throughout the cloud. The spread seems to be larger for the higher X-ray fluxes, however, this is not resulting from a wider column density range, but is merely caused by the wider range in temperatures from the increased $\mathrm{X}$-ray flux. An interesting feature is that there is a range in densities around $n \sim 10^{5} \mathrm{~cm}^{-3}$ where the temperature lingers around a few $100 \mathrm{~K}$. The cooling process is slowed down here because of LTE effects (thermalization and line trapping) until the densities are high enough so that cooling can start to be effective again through gas-dust coupling.

From the color in the phase diagrams we can infer that most of the gas mass lies at high densities, but for higher X-ray fluxes a lot of mass also lies in the low-density, high-temperature regime. This is the area where the cloud is directly irradiated by $\mathrm{X}$-rays and the cloud evaporates. As a consequence, the cloud size and its mass are reduced by this. This has repercussions for the final SFE. When comparing an isothermal model against the model with the highest X-ray flux, for $\zeta_{\mathrm{CR}}=1$, we see that the SFE is reduced from $9.4 \%$ to $8.2 \%$ for a $10^{7} M_{\odot}$ black hole at $t=3 t_{\mathrm{ff}}$, and from $12.3 \%$ to $7.0 \%$ for a $10^{6} M_{\odot}$ black hole (Figs. 10 and 11). The same behavior is found for higher cosmic ray rates (discussed in Sect. 4.2). Despite some fluctuations caused by the randomness of the system, it is interesting to see that the efficiency generally increases a little with our lowest $\mathrm{X}$-ray flux compared to the isothermal models. It seems that a mild X-ray flux of $5.1 \mathrm{erg} \mathrm{s}^{-1} \mathrm{~cm}^{-2}$ or less actually enhances the SFE. See Table 2 for the list of results. Although gas temperatures are higher and the SFE decreases when going to higher $\mathrm{X}$-ray fluxes, the star-formation rate, $\mathrm{SFR}=\mathrm{dSFE} / \mathrm{d} t$, is still high early on. We can see from the SFE plots that star formation is initiated at about the same time, $t_{\text {onset }} \simeq 0.6 t_{\mathrm{ff}}$, irrespective of X-rays. We find that the quenching of star formation caused by X-ray heating is balanced by the increased densities resulting from the thermal pressure that the radiation field creates. An ionizing pressure front compresses the cloud, which leads to very efficient star formation, similar to, if not more than, the colder, isothermal, cloud models. Figure 1 shows a 2D slice to this effect. 


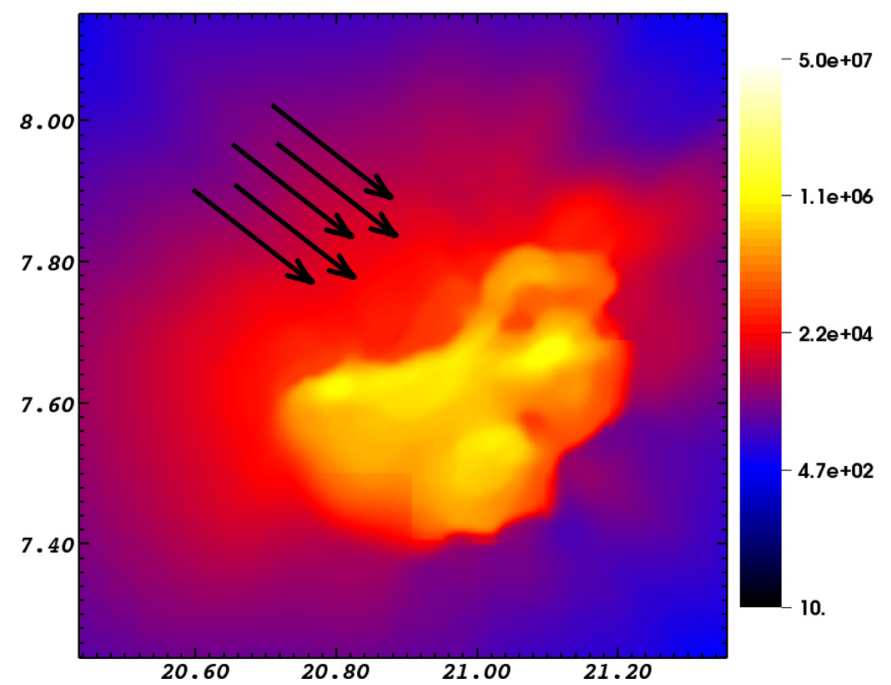

Fig. 1. Density morphology of model M19, i.e., with $F_{X}=$ $160 \mathrm{erg} \mathrm{s}^{-1} \mathrm{~cm}^{-2}$, at $t=2 / 3 t_{\mathrm{ff}}$. The image shows a slice through the $X Y$-plane of the center of the cloud. The color represents the number density $\left(\mathrm{cm}^{-3}\right)$ and the axes are in parsec. The arrows represent the direction of radiation emanating from the black hole, which is located at the upper left side.

We see that the IMFs tend to get flatter for the higher X-ray fluxes. The best power-law fit gives us a nice Salpeter slope with $\Gamma_{\text {powfit }}=-1.34$ for model M 01, i.e., without X-rays, and a slope of $\Gamma_{\text {powfit }}=-1.31,-1.24,-1.01$ for models M 07, M 13, and M 19, i.e., for fixed parameters but with increasing X-ray flux. A lognormal distribution also seems to fit most of the IMFs quite well. The same trend is visible for all other cloud conditions. This implies that the IMF shape depends on the degree to which the cloud is irradiated. Another effect is that the turnover mass shifts toward higher masses with increasing flux, from $M_{\text {turn }}=0.54 M_{\odot}$, at the lowest X-ray flux (M 07), to about 0.70 and $1.51 M_{\odot}$ for the higher fluxes (M 13 and M 19). However, the corresponding isothermal fiducial model (M 01) seems to have a slightly higher turn-over mass, $0.66 M_{\odot}$, than our lowest X-ray flux (5.1 $\mathrm{erg} \mathrm{s}^{-1} \mathrm{~cm}^{-2}$ ) model. Again, a similar trend is seen for all cloud models.

\subsection{Effects of cosmic rays}

Cosmic rays can easily penetrate a molecular cloud and heat the gas uniformally. As such, they set the minimum attainable temperature in these systems (Goldsmith \& Langer 1978; Bergin $\&$ Tafalla 2007). For our model cloud conditions, which have cosmic ray rates of 1,100 , and $3000 \times$ Galactic $\left(\zeta_{\mathrm{CR}, \mathrm{Gal}}=\right.$ $\left.5 \times 10^{-17} \mathrm{~s}^{-1}\right)$, the minimum temperatures are $10 \mathrm{~K}, 50 \mathrm{~K}$, and $200 \mathrm{~K}$ (Papadopoulos et al. 2011; Meijerink et al. 2011). In each of the Figs. 6 to 17 the cosmic ray rate increases from left to right.

In contrast to the X-ray heating, cosmic rays clearly delay the onset of star formation. The first sink particles are formed much later with increasing $\zeta_{\mathrm{CR}}$, see Fig. 2 . The number of sink particles formed is also drastically reduced by up to one order of magnitude, see Table 2. Despite this, once the cloud starts forming stars, it can do so rapidly and massively. The much higher mass each star obtains compensates for the loss in the number of stars. In the end, the SFEs between the different $\zeta_{\mathrm{CR}}$ runs are not much different. This can be explained by the fact that the Jeans mass scales strongly with the temperature and that the cloud fragments into star forming cores that are more massive when the ambient

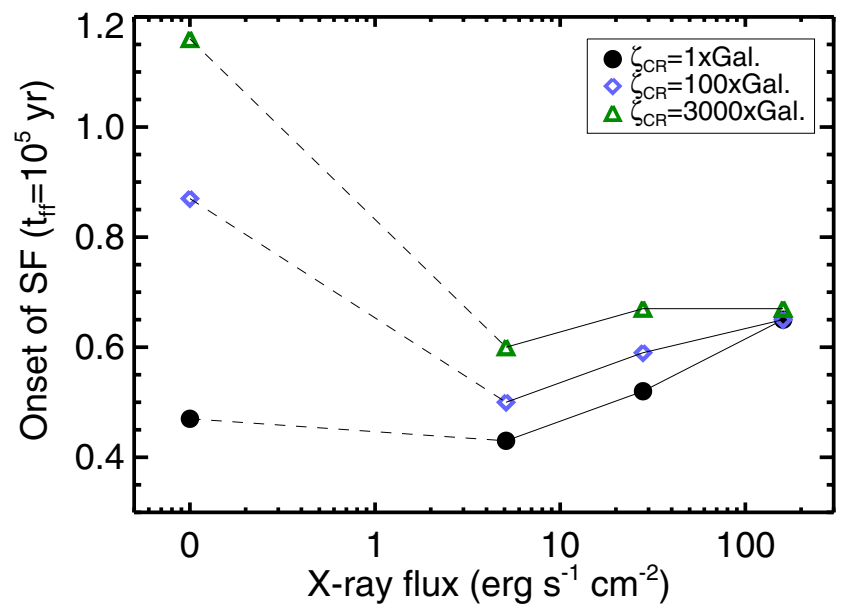

Fig. 2. Onset of star formation for the runs with a $10^{7} M_{\odot}$ black hole. In this figure, the star-formation initiation time is plotted against X-ray flux for three different cosmic ray rates. Higher cosmic ray rates delay the onset of star formation, while increasing the X-ray flux counteracts this. Note that the points on the left side are for the isothermal models that have an X-ray flux of 0 .

temperature is higher. The stars that form out of them are therefore also heavier. These massive stars accrete more matter because there is less competition around, and they tend to grow more. This result also agrees with the idea about fragmentationinduced starvation (Peters et al. 2010; Klessen et al. 2011). But we have a lack of fragmentation in this case and hence very little starvation.

In the absence of X-rays, one obvious result is that high cosmic ray rates strongly inhibit the formation of sub-solar mass sink particles, which follows from the aforementioned Jeans mass argument. The smallest mass we obtained in these runs is about 1 solar mass. This agrees with, and is predicted by, Papadopoulos et al. (2011). From the IMF plots, we see that the effect of cosmic rays on the slope of the mass function is minimal. The lognormal shape is also not much affected, although, given the relatively low number of points at higher $\zeta_{\mathrm{CR}}$ values, one cannot firmly conclude this. Therefore, there is a general shift in the mass distribution toward higher masses. The turnover mass scales toward higher masses as well and has shifted from $0.66 M_{\odot}(\mathrm{M} \mathrm{01})$ to $2.37 M_{\odot}(\mathrm{M} \mathrm{02})$ for the $M_{\mathrm{bh}}=10^{7} M_{\odot}$ runs, and from $0.47 M_{\odot}(\mathrm{M} \mathrm{04})$ to $2.60 M_{\odot}$ (M 05) for the $M_{\mathrm{bh}}=10^{6} M_{\odot}$ runs. This is an increase of a factor of 3.6 and 5.5 , respectively. Between these runs, the Jeans mass increases by a factor of $5^{3 / 2}=11.2(10 \mathrm{~K}$ to $50 \mathrm{~K})$ at the same densities. So, for $\zeta_{\mathrm{CR}}=1$ to 100 times Galactic, it seems that the turn-over mass scales with roughly the temperature rather than the Jeans mass. We found that very massive sink particles were formed in the highest cosmic ray simulations. The most massive sink particle that formed in model M 03 had a mass of 350 solar masses.

\subsection{Effects of UV}

In PDRs, UV radiation heats the gas up to a few thousand $\mathrm{K}$ and will dominate the chemistry of cloud surfaces. The UV radiation can photodissociate molecules, which will lead to the destruction of efficient coolants, and heats through photo-electric emission from (small) dust grains. The UV radiation is strongly attenuated above columns of $N=10^{22} \mathrm{~cm}^{-2}$, at solar metallicity. In our simulations, the model cloud had a starting column of $N=10^{23} \mathrm{~cm}^{-2}$ (center to edge). This grew to $N=1 \times 10^{24} \mathrm{~cm}^{-2}$, and beyond, when collapse was initiated and the first stars were 
Table 2. List of results for each model.

\begin{tabular}{|c|c|c|c|c|c|c|}
\hline Model & IMF slope & $\begin{array}{c}\text { Turn-over mass } \\
{\left[M_{\odot}\right]}\end{array}$ & $\begin{array}{l}\text { Onset of SF } \\
{\left[t_{\mathrm{ff}}=10^{5} \mathrm{yr}\right]}\end{array}$ & $\begin{array}{c}\text { Final SFE } \\
{\left[M_{\text {part. }} / M_{\text {tot. }}\right]}\end{array}$ & Accreted fraction & Number of particles \\
\hline M01 & -1.34 & 0.66 & 0.47 & 0.094 & 0.606 & 247 \\
\hline M02 & -1.44 & 2.37 & 0.87 & 0.092 & 0.691 & 110 \\
\hline M03 & ${ }^{a} a$ & $4.25^{a}$ & 1.16 & 0.089 & 0.747 & 27 \\
\hline M04 & -1.30 & 0.47 & 0.35 & 0.123 & 0.801 & 262 \\
\hline M 05 & -1.24 & 2.60 & 0.67 & 0.125 & 0.795 & 63 \\
\hline М 06 & $-^{a}$ & $5.95^{a}$ & 0.99 & 0.117 & 0.725 & 21 \\
\hline М 07 & -1.31 & 0.54 & 0.43 & 0.099 & 0.609 & 350 \\
\hline M 08 & -1.08 & 0.65 & 0.50 & 0.095 & 0.693 & 227 \\
\hline M 09 & -1.07 & 0.65 & 0.60 & 0.077 & 0.653 & 211 \\
\hline M 10 & -1.04 & 0.43 & 0.36 & 0.125 & 0.768 & 276 \\
\hline M 11 & -1.05 & 0.52 & 0.46 & 0.117 & 0.708 & 137 \\
\hline M 12 & -0.78 & 0.26 & 0.52 & 0.116 & 0.762 & 104 \\
\hline M 13 & -1.24 & 0.70 & 0.52 & 0.102 & 0.607 & 286 \\
\hline M 14 & -1.02 & 0.73 & 0.59 & 0.092 & 0.597 & 211 \\
\hline M 15 & -0.92 & 0.77 & 0.67 & 0.093 & 0.619 & 171 \\
\hline M 16 & -1.16 & 0.68 & 0.42 & 0.104 & 0.598 & 202 \\
\hline M 17 & -1.01 & 0.65 & 0.52 & 0.100 & 0.545 & 153 \\
\hline M 18 & -0.58 & 0.52 & 0.59 & 0.094 & 0.592 & 104 \\
\hline M 19 & -1.01 & 1.51 & 0.65 & 0.082 & 0.637 & 138 \\
\hline M 20 & -0.91 & 1.62 & 0.65 & 0.076 & 0.620 & 134 \\
\hline M 21 & -0.85 & 1.43 & 0.67 & 0.073 & 0.621 & 111 \\
\hline M 22 & -0.82 & 1.12 & 0.55 & 0.070 & 0.647 & 80 \\
\hline M 23 & -0.70 & 1.91 & 0.57 & 0.071 & 0.623 & 79 \\
\hline M24 & -0.62 & 1.36 & 0.62 & 0.073 & 0.627 & 58 \\
\hline M 25 & -1.32 & 1.20 & 0.74 & 0.014 & 0.476 & 84 \\
\hline M26 & $-^{a}$ & $-^{a}$ & 0.80 & 0.001 & 0.681 & 3 \\
\hline M 27 & $-{ }^{b}$ & $-{ }^{b}$ & $-b$ & $-{ }^{b}$ & $-{ }^{b}$ & 0 \\
\hline M 28 & -1.30 & 1.62 & 0.66 & 0.037 & 0.637 & 108 \\
\hline M 29 & -1.28 & 1.86 & 0.66 & 0.024 & 0.697 & 59 \\
\hline M30 & -1.07 & 1.53 & 0.65 & 0.013 & 0.618 & 49 \\
\hline M 31 & -1.48 & 0.55 & 0.49 & 0.103 & 0.638 & 262 \\
\hline M 32 & -1.31 & 2.30 & 0.90 & 0.099 & 0.728 & 106 \\
\hline M33 & $-^{a}$ & $4.30^{a}$ & 1.16 & 0.093 & 0.850 & 19 \\
\hline M 34 & -1.23 & 0.43 & 0.40 & 0.101 & 0.568 & 332 \\
\hline M 35 & -0.99 & 0.57 & 0.54 & 0.085 & 0.658 & 230 \\
\hline M 36 & -1.06 & 0.65 & 0.60 & 0.096 & 0.638 & 198 \\
\hline M 37 & -1.05 & 0.59 & 0.51 & 0.107 & 0.650 & 281 \\
\hline M 38 & -1.04 & 0.66 & 0.56 & 0.101 & 0.647 & 207 \\
\hline M39 & -0.99 & 0.88 & 0.67 & 0.101 & 0.748 & 163 \\
\hline M 40 & -1.40 & 1.58 & 0.66 & 0.084 & 0.641 & 142 \\
\hline M 41 & -1.06 & 1.47 & 0.66 & 0.082 & 0.648 & 132 \\
\hline M 42 & -0.79 & 1.52 & 0.67 & 0.069 & 0.614 & 103 \\
\hline
\end{tabular}

Notes. ${ }^{(a)}$ Too few particles have formed here to properly construct an IMF. ${ }^{(b)}$ No particles have formed in this simulation.

about to form. Hence, it is not expected that UV radiation will have a direct effect on the results. Only by heating the low density regions, $n<10^{4} \mathrm{~cm}^{-3}$, and by pressurizing the medium where the molecular cloud is embedded in, the results might be influenced. We assume that there is a uniform background UV field. The results for the model clouds in UV-dominated environments are shown in Figs. 9, 13, and 17.

We chose to simulate the models with increased UV radiation for a single black hole mass, $M_{\mathrm{bh}}=10^{7} M_{\odot}$, as can be seen from the table (Table 1). The reason was that our initial studies immediately showed that the direct effect of an external UV radiation field on our model clouds was modest. Follow-up simulations confirmed that the column density $\left(>10^{22} \mathrm{~cm}^{-2}\right)$ of the molecular cloud was, as predicted, already too high for UV radiation to penetrate even the edge of the cloud $(<0.01 \mathrm{pc})$ and that turbulent effects and other instabilities did not provide the opportunity for UV radiation to do much damage. When X-rays were present, the gas heating was dominated by them. This is a consequence of the much higher (10-50\%) ionization heating efficiency compared to photo-electric heating $(0.1-1 \%)$ by UV irradiated dust grains. However, UV radiation does heat the lowdensity gas, therefore, it influences the late-time accretion.

The phase diagrams for the UV runs confirm that the radiation does not penetrate the natal cloud beyond a few magnitudes of visual extinction at one free-fall time. At low densities, we see that UV heats the gas to several hundred and upto a few thousand $\mathrm{K}$. Above $10^{4} \mathrm{~cm}^{-3}$ it has no impact. In the models together with X-rays, even with the lowest X-ray flux $F_{\mathrm{X}}=5.1 \mathrm{erg} \mathrm{s}^{-1} \mathrm{~cm}^{-2}$, there is little difference in the phase diagrams compared to the non-UV runs. Only the low-density $\leq 10^{4} \mathrm{~cm}^{-3}$, unshielded (in the UV) regions are heated up by UV. We also see that the SFE plots are not much different from their UV deficient counterparts. The slope of the IMF, on the other hand, is more sensitive to variations. We see that massive stars accrete less matter during the late stages of the run, i.e., $t \gtrsim 2 t_{\mathrm{ff}}$, because the temperature is higher at low densities. This 

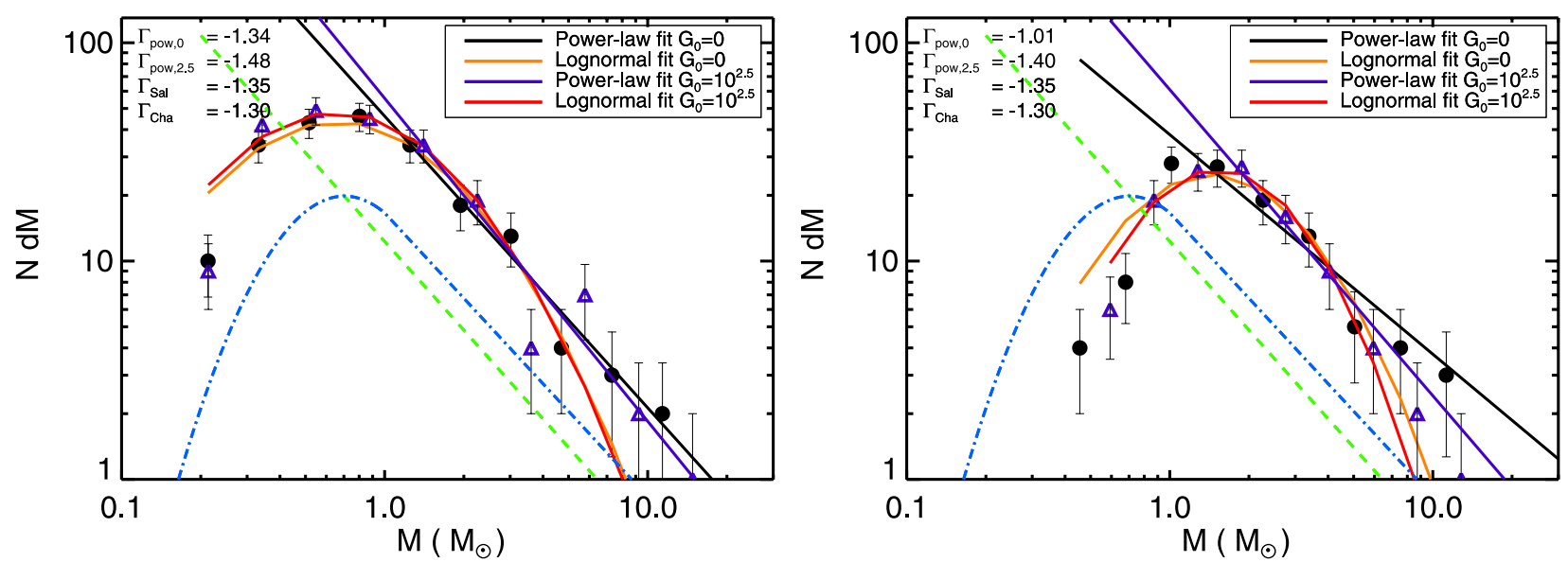

Fig. 3. Comparison of IMFs of the UV runs against their non-UV counterparts. The left panel shows the IMFs of the models M 01 and M 31 (no X-rays). The right panel shows the IMFs of the models M 19 and M 40 (highest X-ray flux). For comparison purposes, the Salpeter IMF (green dashed line) and the Chabrier IMF (blue dot-dashed line) are shown as fitted to our fiducial case.

while most of the late-time accretion normally comes from the low-density, cold regions. Especially for the highest $F_{\mathrm{X}}$ runs, the slope of the IMF becomes slightly steeper because of a decrease in the mass of the most massive stars in the presence of a UV background. In Fig. 3 we plot the IMFs for four model runs, M 01 versus M 31 and M 19 versus M 40, that is, with and without UV, together in the same figure to highlight this effect.

\subsection{Effects of shear}

If there is a strong gravity, the gravitational pull on each side of a bound extended object will cause a differential acceleration. If the kinetic energy resulting from the velocity difference is close to or on the order of the gravitational binding energy of the object, it will play an important role in the dynamics of the cloud. The object will be torn apart if the corresponding kinetic energy is much higher than its binding energy.

Our model cloud has a binding energy of

$E_{\text {bind }}=\frac{3 G M_{\text {cloud }}^{2}}{5 r_{\text {cloud }}}=1.02 \times 10^{47} \mathrm{erg}$,

where $M_{\text {cloud }}$ and $r_{\text {cloud }}$ are the cloud mass and radius. The maximum shear for the models with the three different black hole masses for a cloud that is at $10 \mathrm{pc}$ distance results in kinetic energies of $E_{\text {shear }}=\left[4 \times 10^{45}, 4 \times 10^{46}, 4 \times 10^{47}\right] \mathrm{erg}$, with $\Delta \mathrm{v}_{\text {shear }}=[0.7,2.2,7.0] \mathrm{km} \mathrm{s}^{-1}$. The velocity difference resulting from the gravitational stresses of the two higher black hole masses, $M_{\mathrm{bh}}=\left[10^{7}, 10^{8}\right] M_{\odot}$, is on the order of the applied initial turbulence $\left(5 \mathrm{~km} \mathrm{~s}^{-1}\right)$, and will therefore play an important role in the evolution of the cloud. Whereas the velocity shear caused by a black hole with $M_{\mathrm{bh}}=10^{6} M_{\odot}$ will have very little impact on the dynamics of the simulation. Figures 6, 10, and 14 display the models with $M_{\mathrm{bh}}=10^{7} M_{\odot}$. Figures 7, 11, and 15 display the models with $M_{\mathrm{bh}}=10^{6} M_{\odot}$. While Figs. 8, 12 , and 16 show the models with $M_{\mathrm{bh}}=10^{8} M_{\odot}$.

From the phase diagrams we infer that the column density range is more extended for the lower shear runs. A salient feature is the dual phase structure that can be seen in the phase diagrams at low densities. This is because the cloud retains its size and shape for a longer period of time when there is less shear, developing a wide column density range for the same densities. Moreover, we see from the density images that the cloud becomes more asymmetric with increasing $M_{\mathrm{bh}}$. The cloud is stretched in the direction of the orbit but compressed perpendicular to it, see Fig. 4. Owing to their morphological effect on the cloud, the gravitational stresses are the cause for the reduced column density range. This is the reason why in the presence of $\mathrm{X}$-rays a larger surface is directly irradiated by radiation with less attenuation throughout the cloud.

We find that the final SFEs are reduced for higher black hole masses. Furthermore, we see that the star-formation rate has dropped as well, while the onset of star formation is delayed with increasing shear. However, when we look at the numbers of sink particles formed, we find that they are remarkably higher for large shear, that is, comparing $M_{\mathrm{bh}}=10^{7} M_{\odot}$ against $10^{6} M_{\odot}$. The simulations with the highest shear, $M_{\mathrm{bh}}=10^{8} M_{\odot}$, strongly inhibit the formation of all stars. See Table 2 for details. This increase in the number of sink particles with a stable SFE shows that the formation of low-mass stars is favored for the case with $M_{\mathrm{bh}}=10^{7} M_{\odot}$. When we look at the IMF plots, we do see that for the higher shear runs the mass function generally comprises more low-mass stars and fewer high-mass stars. This result agrees with gravoturbulent fragmentation (Klessen \& Ballesteros-Paredes 2004).

\subsection{Accretion onto sink particles}

Generally most of the mass growth of the sink particles comes from accretion. Proto-stars gain about $66 \%$ of their mass in this way. This gives us an average accretion rate of $\sim 10^{-5} M_{\odot} / y$. Owing to the restricted time resolution of our checkpoint files, immediate merging of newly created sink particles within $1 / 30$ th of a free-fall time $\left(\sim 3 \times 10^{3} \mathrm{yr}\right)$, is considered as accretion. However, this should not occur very often because only about $15 \%$ of the particles merge during a run and this is spread throughout the whole simulation. The fraction of accreted mass at $t=3 t_{\mathrm{ff}}$ is, on average, $\bar{f}_{\mathrm{accr}} \simeq 0.683$ when there is little black hole shear $\left(M_{\mathrm{bh}}=10^{6} M_{\odot}\right)$. This is reduced to $\bar{f}_{\text {accr }} \simeq 0.642$ when we increase $M_{\mathrm{bh}}$ to $10^{7} M_{\odot}$. Similarly, when we increase the X-ray flux from $0,5.1,28$ to 160 , the accreted mass fraction drops from $0.774,0.746,0.579$ to 0.632 for $M_{\mathrm{bh}}=10^{6} M_{\odot}$ and from $0.681,0.652,0.608$ to 0.626 for $M_{\mathrm{bh}}=10^{7} M_{\odot}$. See Table 2 for a complete list of accreted mass fractions. Note that the highest X-ray flux has a slightly higher mass accretion fraction than the second highest flux. This may be a consequence of the interplay between reduced accretion caused by the increase in temperature, as sound speed scales with $T^{1 / 2}$, and the increase 

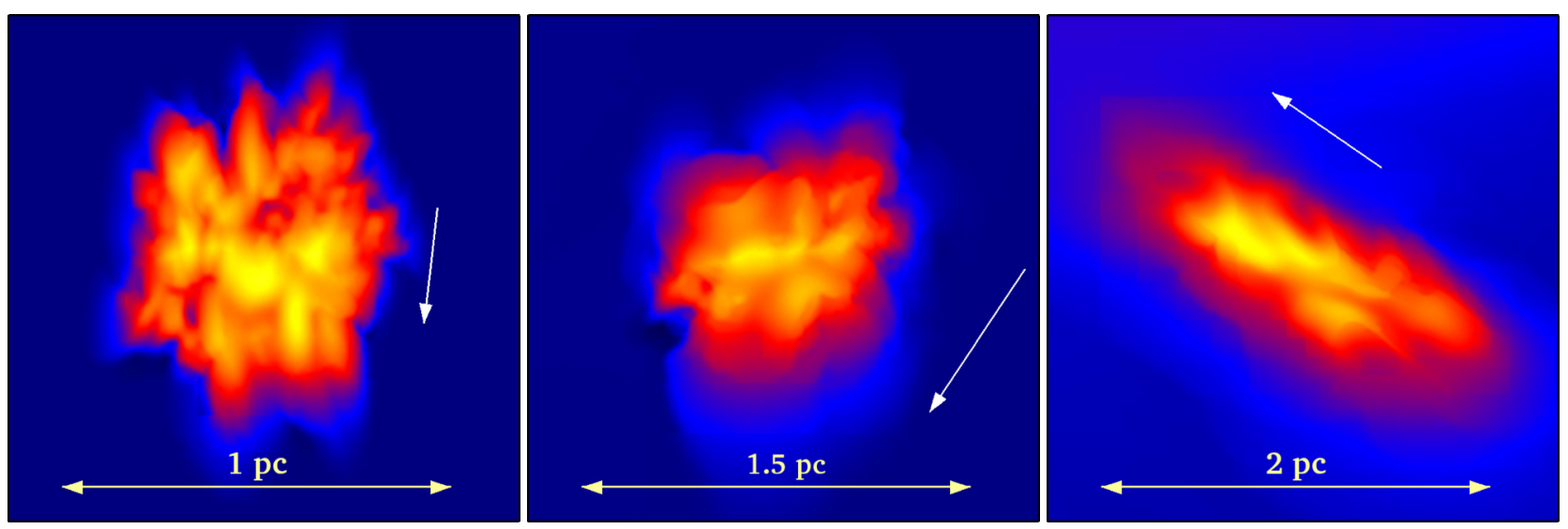

Fig. 4. Density slices through the center of the cloud at $t=2 / 3 t_{\mathrm{ff}}$ for three different black hole mass models. The clouds are stretched in the direction of motion with increasing black hole mass, but compressed in the direction perpendicular to this. The black hole masses are $10^{6} M_{\odot}$ for model M04 (left panel), $10^{7} M_{\odot}$ for model M 01 (middle panel), and $10^{8} M_{\odot}$ for model M 25 (right panel). The white arrow shows the direction of the orbital motion.

in accretion caused by the larger gas reservoir available because of the lower SFE. Apparently, the balance tips towards more accretion at the highest X-ray flux. The fractions for the runs with the highest shear are too volatile to consider because very little star formation has occurred in these runs.

From Eq. (5) we see that the accretion rate scales inversely with the sound speed and gas velocity to the third power. This is a strong scaling and is the dominant factor for the decrease in accretion as gas turbulence increases. The strong differential velocities caused by shear from the black hole cascade down to smaller scales and yield turbulence. The turbulence still decays in time, but the decay is less rapid compared to when there is less shear. Thermal pressure as a consequence of the increased temperatures for the X-ray models also contributes to higher velocities and decreases the accretion rate. We do not see a clear correlation between accretion and cosmic rays, though. There can be two reasons for this. First of all, there is no gradient in pressure throughout the cloud because of the increase in cosmic ray ionization rate (they heat all gas uniformly). Second, the heating by cosmic rays is relatively modest, yielding much lower temperatures than that of UV or X-ray heated gas.

For the UV runs, we fond notable differences after two freefall times. Owing to UV heating, accretion onto sink particles from the low density environment, $n<10^{4} \mathrm{~cm}^{-3}$, is drastically reduced. This is normally the main source for particles to gain mass at the later stages of the simulation. The more massive sink particles are also more strongly affected. Consequently, the models that produced a top-heavy IMF (these are the runs with increased X-ray flux) are restrained. A slightly steeper IMF slope is the result. The turn-over mass and the SFEs, on the other hand, are not significantly affected.

\section{Conclusions and discussion}

By means of numerical simulations, we have performed an extensive study on the effects of various types of irradiation (X-rays, cosmic rays, and UV) and shear on collapsing molecular clouds in active galactic environments. We analyzed how the star-formation efficiencies and the initial mass functions are affected for 42 different cloud models. Our general result is that the IMF deviates from a Salpeter shape in extreme environments. Still, for a mixture of ambient conditions, their effects on the mass function are somewhat balanced and a deviation from a Salpeter shape is less pronounced. Here, we evaluate the differences and similarities in detail. All quantifiable results are listed in Table 2.

The IMF nicely follows a lognormal distribution with a Salpeter slope for the isothermal runs. Our fiducial model M 01 and model M 04, which have similar conditions to those of the Milky Way, agree well with a Chabrier IMF and match a Salpeter slope excellently, as can be seen in Fig. 14. Increasing the cosmic ray rate or the black hole shear did not change the slope of the mass function by much, except perhaps for the highest $\zeta_{\mathrm{CR}}$ and the highest $M_{\mathrm{bh}}$. Owing to the insufficient number of sink particles, it was not possible to fit these cases well.

The interplay between X-ray, shear and cosmic rays is as follows. The power-law slope of the IMF flattens and becomes non-Salpeter when we increased the X-ray flux from 0 to $160 \mathrm{erg} \mathrm{s}^{-1} \mathrm{~cm}^{-2}$. We found that the slope drops from around $\Gamma_{\text {powfit }}=-1.35\left(F_{\mathrm{X}}=0\right)$ to about $\Gamma_{\text {powfit }}=-1\left(F_{\mathrm{X}}=160\right)$ for the runs with $M_{\mathrm{bh}}=10^{7} M_{\odot}$, and to about $\Gamma_{\text {powfit }}=-0.8\left(F_{\mathrm{X}}=160\right)$ when $M_{\mathrm{bh}}=10^{6} M_{\odot}$. We saw that the flattening is less prominent when the shear is stronger. We attributed this effect to the change in shape of the cloud, see Fig. 4. When gravitational shear is stronger, the irradiated face of the cloud is larger owing to a stretching of the cloud in the direction of the orbit. A bigger region is thus compressed by thermal pressure. We also saw that the cloud obtains a rotational motion. Its rotational time is comparable to, though slightly faster than, the orbital time. This rotation is slow, but it does cause the regions that were initially hot to become shielded from X-rays. These regions, whose densities were raised by thermal compression now cooled down quickly in the shade and formed low-mass stars easily. This while the model clouds in the lowest $M_{\mathrm{bh}}=10^{6} M_{\odot}$ runs face the radiation source on the same side for a much longer period because their orbital time is about three times longer. This argument is supported by the behavior of the models with the highest shear. For these, the isothermal cosmic ray runs do not form sink particles at all, but in the presence of X-rays, star formation does still occur thanks to compression. Cosmic rays, on the other hand, enhance the flattening of the power-law slope. At the highest $\zeta_{\mathrm{CR}}$, the IMF slope drops further from about $\Gamma_{\text {powfit }}=-1$ to -0.8 for the $M_{\mathrm{bh}}=10^{7} M_{\odot}$ runs, and from $\Gamma_{\text {powfit }}=-0.8$ to -0.6 for the $M_{\mathrm{bh}}=10^{6} M_{\odot}$ runs. We can understand this behavior because there are regions in the cloud that are shielded from X-rays and where the gas behaves in an isothermal manner. These places will form more massive stars because of the 
higher temperatures. Because we plotted all stars together in the IMF plot, a steep overall IMF slope was created.

We found that the turn-over mass shifts towards higher masses for increasing X-ray fluxes and cosmic ray rates. A simple Jeans mass argument is sufficient to explain this behavior. Because the Jeans mass strongly depends on the temperature (Eq. (3)), and this sets the minimum mass for the fragmentation scale, stars that form in massive cores tend to be more massive as well. The effect of cosmic rays is less prominent in the presence of X-rays because of the softer equation of state, as mentioned above. This, in the end, makes the turn-over mass less strongly dependent on the Jeans mass, and in part explains the constancy of the characteristic mass in many star-forming regions (Elmegreen et al. 2008; Bate 2009).

$\mathrm{X}$-rays reduce the final SFE by up to $40 \%$ in the presence of a strong X-ray flux, $F_{X}=160 \mathrm{erg} \mathrm{s}^{-1} \mathrm{~cm}^{-2}$. This effect is very modest for the lowest X-ray flux, $F_{X}=5.1 \mathrm{erg} \mathrm{s}^{-1} \mathrm{~cm}^{-2}$. Indeed, there is a slight increase in the SFE for the lower cosmic ray rates. For $\mathrm{X}$-rays to have any significant impact on to the efficiency of star formation, a flux of at least $F_{\mathrm{X}} \geq 5.1 \mathrm{erg} \mathrm{s}^{-1} \mathrm{~cm}^{-2}$ is needed. The main reason for the reduced efficiency is that the cloud evaporates from the irradiated side, thereby reducing the mass of the molecular cloud. This, however, also increases the densities at the same side due to an ionizing compression front such that the star-formation rate remains high.

We also saw a reduction in formation efficiency when we increased the black hole shear. We achieved this by increasing the black hole mass while keeping the distance of the cloud fixed at $10 \mathrm{pc}$, thereby changing the $M_{\mathrm{bh}} / \mathrm{d}_{\mathrm{bh}}^{3}$ ratio. For the runs with the highest shear, $M_{\mathrm{bh}}=10^{8} M_{\odot}$, star formation was almost completely quenched. In this case, the kinetic energy from the velocity divergence that the black hole injects is greater than the binding energy of the cloud. However, we found that stars can still form when thermal compression by X-rays enhances cloud collapse, albeit with very low efficiency. The effect of shear on star formation is also more dramatic than that of the X-rays. Besides reducing the efficiency, the rate at which stars form is also affected. From the shape of the curves of the SFE plots in Figs. 10 to 13, we saw that the increase in SFE in time was less steep and somewhat more irregular for higher $M_{\mathrm{bh}}$. The cause for this effect is the enhancement of the turbulence from the shearing motion cascading down to smaller scales. We saw that the turbulence remained strong, $F W H M=4-5 \mathrm{~km} \mathrm{~s}^{-1}$ on the scale of the cloud at one free-fall time, for the $M_{\mathrm{bh}}=10^{7}$ and $10^{8} M_{\odot}$ runs, while the turbulence decayed to about $1 \mathrm{~km} \mathrm{~s}^{-1}$ for $M_{\mathrm{bh}}=10^{6} M_{\odot}$. In addition to this, stars start to form later with increasing shear.

Cosmic rays also delay the onset of star formation. Interestingly, the SFE and the star-formation rate were not affected. However, the number of sink particles was drastically reduced with increasing $\zeta_{\mathrm{CR}}$. The average mass per sink particle, on the other hand, rose comparably, while the formation of low-mass stars was strongly inhibited. There was a significant side-effect from X-rays that counteracts this. When we considered the difference between the isothermal runs and the X-ray runs for any $\zeta_{\mathrm{CR}}$, we saw that many more sink particles were formed when there was an X-ray source. Especially the number of low-mass stars was higher for the X-ray runs. This can be inferred form the shape of the mass function in Figs. 14 to 17. One would normally expect that because of the increased gas temperatures from X-ray heating, star formation would be strongly suppressed for the same cosmic ray rate. However, the equation of state plays a crucial role here. Despite the higher temperatures, the compressibility of the gas, $\gamma$ being less than unity, caused the molecular cloud to fragment more easily. This while the cosmic ray runs enjoyed an isothermal, $\gamma=1$, equation of state. Therefore, low-mass stars are able to form with much less effort. The delay in star formation caused by cosmic ray heating was also counterbalanced in the presence of X-rays. This effect was more prominent with increasing $\zeta_{\mathrm{CR}}$, see Fig. 2. A time difference in the onset of star formation of more than one-third free-fall time $\left(>3.3 \times 10^{4} \mathrm{~s}\right)$ could be seen for models with $\zeta_{\mathrm{CR}}=3000 \times$ Galactic.

For our models with UV, we found that it has only a modest impact on the results. This was expected because our model cloud had a column of $N \simeq 10^{23} \mathrm{~cm}^{-2}$ at the start of the simulation, and increased its (column) density with time. UV radiation is strongly attenuated above columns of $N=10^{22} \mathrm{~cm}^{-2}$, for solar metallicity. We presented the models with UV for $M_{\mathrm{bh}}=10^{7} M_{\odot}$ and $F_{\mathrm{UV}}=10^{2.5} G_{0}$ only, to asses whether increased external pressure and the higher cloud edge gas temperatures were able to play an important role in the evolution of the cloud. We found that by increasing the temperature for the lower densities, $n<10^{4} \mathrm{~cm}^{-3}$, accretion onto particles was reduced at later times, i.e., $t \gtrsim 2 t_{\mathrm{ff}}$. This impacted the growth of the massive particles later on. As such, the slope of the IMF, especially in the presence of X-rays, is somewhat more steep, in general. However, we found that the change in the SFEs were almost negligible when we considered an isotropic UV field with a strength of $10^{2.5} \mathrm{G}_{0}$, as compared to the non-UV runs. For cloud conditions such as in this work, we found that UV radiation can still be of importance to the formation of stars through secondary channels.

Accretion rates onto point particles in a turbulent medium may be different from the rates (Eq. (7)) used in this work, as Krumholz et al. (2006) point out. Our medium was close to homogeneous on scales of a few parsec. On the other hand, outflows and jets from young stars could reduce the net accretion rates on smaller scales. Therefore, using a homogeneous flow approximation might overestimate the accretion rates and we consider them upper limits. In addition to this, Stamatellos et al. (2011) show that episodic accretion for low-mass stars with bursts of radiative feedback can also affect the fragmentation properties. We did not consider such effects. We compared our model results against each other, however. In this way, our findings are less sensitive to the above mentioned problems, and relative changes in the IMF are well defined.

The velocity shear through the cloud caused by black hole gravity decreased as the cloud contracted. With time, the model cloud in our simulations shrank in the direction perpendicular to the orbit owing to self-gravity and turbulent motions. On average, the model cloud shrank by a factor of two, at most in one free-fall time. This reduced the shear by the same amount as $\Delta v$ scales with $r_{\text {cloud }}$. Thus, the injected energy decreased with time, but not strongly.

The maximum resolution allowed was $4096^{3}$ cells. This in itself is quite a good resolution, and to follow it for long dynamical timescales, like we do, makes it difficult to go much higher. Because we used an adaptive grid, it was more important to properly resolve the grid dynamically. A proper means to do so was to resolve the Jeans length adequately everywhere in time and space. Truelove et al. (1997) say that the Jeans length should be resolved by at least four cells, but in the case of (M)HD simulations in magnetic fields, a minimum resolution of 30 cells is required according to Federrath et al. (2011). We resolved the Jeans length in our simulations by at least 10 cells. This means that the minimum resolution always lay somewhere between 10-20 cells. However, our resolution criterion was so strict that we resolved a whole block of 512 cells once even a single cell 


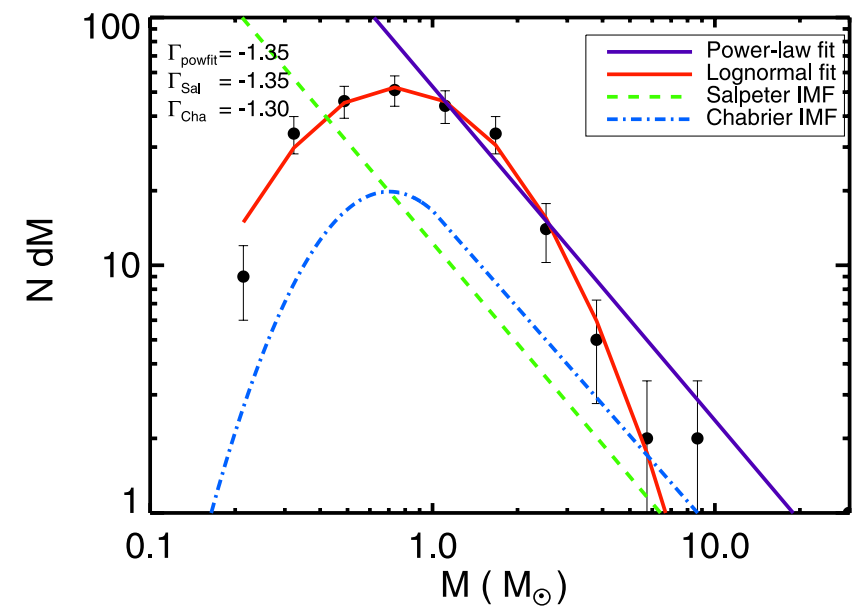

Fig. 5. IMF of model M01 without sink particle merging. In this image, the Salpeter IMF (green dashed) and the Chabrier IMF (blue dotdashed) are displayed as fitted to our fiducial model. A linear fit and a lognormal fit are shown as purple and red lines.

came close to the minimum of 10 cells, while we only de-refined if all the cells within the block had stretched beyond 25 times the Jeans length. This in effect makes the general resolution in our runs much higher than the 10 cells that we mention, and approximately about $50-60$ cells on average.

Stellar multiplicity was not covered in this work and binaries do not form naturally from the sink particle routine. To achieve that, cloud collapse should be followed to the highest grid resolutions to resolve the gas dynamics properly and allow protostars to form. This is very difficult to perform numerically. If we were to consider the sink particles in our work as binaries, then the found shapes of the IMFs would not be different, and would only be rescaled to lower masses. As a test, we re-ran two of our simulations without sink particle merging. We found that the merging routine does not influence the results much. Figure 5 shows a plot of model M 01 without merging of sink particles.

In the future, we intend to perform studies for conditions much closer to the black hole, i.e., within $1 \mathrm{pc}$ of galactic nuclei, to model massive stars as observed in Sgr A* and in M 31. We also plan to incorporate our results into simulations of AGN evolution and intend to look into the stability of the entire disk.

Acknowledgements. We are grateful to the anonymous referee for an insightful and constructive report that helped to improve this work. We would also like to thank Dominik R.G. Schleicher for useful discussions on the behavior of the IMF in active galaxies. S.H. thanks M. A. Latif for insightful comments on the numerical part of this work and P. Papadopoulos for his eager input on cosmic rays. The FLASH code was in part developed by the DOE-supported Alliance Center for Astrophysical Thermonuclear Flashes (ACS) at the University of Chicago. The simulations were run on the dedicated special purpose machines "Gemini" at the Kapteyn Astronomical Institute, University of Groningen and at the Donald Smits Center for Information Technology (CIT) using the Millipede Cluster, University of Groningen.

\section{References}

Aalto, S. 2005, Ap\&SS, 295, 143

Alexander, R. D., Armitage, P. J., Cuadra, J., \& Begelman, M. C. 2008, ApJ, 674,927

Alig, C., Burkert, A., Johansson, P. H., \& Schartmann, M. 2011, MNRAS, 412, 469

Aykutalp, A., \& Spaans, M. 2011, ApJ, 737, 63

Ballero, S. K., Kroupa, P., \& Matteucci, F. 2007, A\&A, 467, 117

Ballero, S. K., Matteucci, F., Ciotti, L., Calura, F., \& Padovani, P. 2008, A\&A, 478,335

Bartko, H., Martins, F., Trippe, S., et al. 2010, ApJ, 708, 834

Bastian, N., Covey, K. R., \& Meyer, M. R. 2010, ARA\&A, 48, 339
Bate, M. R. 2009, MNRAS, 392, 1363

Bate, M. R. 2010, MNRAS, 404, L79

Bate, M. R., Bonnell, I. A., \& Price, N. M. 1995, MNRAS, 277, 362

Baugh, C. M., Lacey, C. G., Frenk, C. S., et al. 2005, MNRAS, 356, 1191

Bergin, E. A., \& Tafalla, M. 2007, ARA\&A, 45, 339

Binns, W. R., Wiedenbeck, M. E., Arnould, M., et al. 2008, New A Rev., 52, 427

Bondi, H. 1952, MNRAS, 112, 195

Bondi, H., \& Hoyle, F. 1944, MNRAS, 104, 273

Bonnell, I. A., \& Rice, W. K. M. 2008, Science, 321, 1060

Brassington, N. J., Ponman, T. J., \& Read, A. M. 2007, MNRAS, 377, 1439

Caselli, P., Benson, P. J., Myers, P. C., \& Tafalla, M. 2002, ApJ, 572, 238

Chabrier, G. 2003, PASP, 115, 763

Clark, P. C., Glover, S. C. O., Bonnell, I. A., \& Klessen, R. S. 2009 [arXiv:0904.3302]

Clark, P. C., Glover, S. C. O., Smith, R. J., et al. 2011, Science, 331, 1040

Colella, P., \& Woodward, P. R. 1984, J. Comp. Phys., 54, 174

Dabringhausen, J., Kroupa, P., \& Baumgardt, H. 2009, MNRAS, 394, 1529

Davé, R. 2008, MNRAS, 385, 147

Dubey, A., Antypas, K., Ganapathy, M. K., et al. 2009, Parallel Computing, 35, 512

Elmegreen, B. G. 2009, in The Evolving ISM in the Milky Way and Nearby Galaxies

Elmegreen, B. G., Klessen, R. S., \& Wilson, C. D. 2008, ApJ, 681, 365

Espinoza, P., Selman, F. J., \& Melnick, J. 2009, A\&A, 501, 563

Falgarone, E., Pety, J., \& Phillips, T. G. 2001, ApJ, 555, 178

Federrath, C., Klessen, R. S., \& Schmidt, W. 2009, ApJ, 692, 364

Federrath, C., Banerjee, R., Clark, P. C., \& Klessen, R. S. 2010, ApJ, 713, 269

Federrath, C., Sur, S., Schleicher, D. R. G., Banerjee, R., \& Klessen, R. S. 2011, ApJ, 731, 62

Figer, D. F. 2005a, Nature, 434, 192

Figer, D. F. 2005b, in The Initial Mass Function 50 Years Later, ed. E. Corbelli, F. Palla, \& H. Zinnecker, Astrophys. Space Sci. Libr., 327, 89

Frieswijk, W. W. F., Spaans, M., Shipman, R. F., Teyssier, D., \& Hily-Blant, P. 2007, A\&A, 475, 263

Genzel, R., Schödel, R., Ott, T., et al. 2003, ApJ, 594, 812

Girichidis, P., Federrath, C., Banerjee, R., \& Klessen, R. S. 2011, MNRAS, 413, 2741

Goldsmith, P. F., \& Langer, W. D. 1978, ApJ, 222, 881

Habing, H. J. 1968, Bull. Astron. Inst. Netherlands, 19, 421

Heyer, M. H., \& Brunt, C. M. 2004, ApJ, 615, L45

Hobbs, A., \& Nayakshin, S. 2009, MNRAS, 394, 191

Hocuk, S., \& Barthel, P. D. 2010, A\&A, 523, A9

Hocuk, S., \& Spaans, M. 2010a, A\&A, 522, A24

Hocuk, S., \& Spaans, M. 2010b, A\&A, 510, A110

Hollenbach, D. J., \& Tielens, A. G. G. M. 1999, Rev. Modern Phys., 71, 173

Hsu, W.-H., Hartmann, L., Heitsch, F., \& Gómez, G. C. 2010, ApJ, 721, 1531

Hughes, D. H., Serjeant, S., Dunlop, J., et al. 1998, Nature, 394, 241

Jappsen, A., Klessen, R. S., Larson, R. B., Li, Y., \& Mac Low, M. 2005, A\&A, 435,611

Klessen, R. S. 2001, ApJ, 556, 837

Klessen, R. S., \& Ballesteros-Paredes, J. 2004, Bal. Astron., 13, 365

Klessen, R. S., Ballesteros-Paredes, J., Vázquez-Semadeni, E., \& Durán-Rojas, C. 2005a, ApJ, 620, 786

Klessen, R. S., Spaans, M., \& Jappsen, A.-K. 2005b, in Massive Star Birth: A Crossroads of Astrophysics, ed. R. Cesaroni, M. Felli, E. Churchwell, \& M. Walmsley, IAU Symp., 227, 337

Klessen, R. S., Spaans, M., \& Jappsen, A.-K. 2007, MNRAS, 374, L29

Klessen, R. S., Peters, T., Banerjee, R., et al. 2011, in IAU Symp. 270, ed.

J. Alves, B. G. Elmegreen, J. M. Girart, \& V. Trimble, 107

Kroupa, P. 2001, MNRAS, 322, 231

Krumholz, M. R. 2011, in AIP Conf. Ser., 1386, ed. E. Telles, R. Dupke, \&

D. Lazzaro, 9

Krumholz, M. R., McKee, C. F., \& Klein, R. I. 2004, ApJ, 611, 399

Krumholz, M. R., McKee, C. F., \& Klein, R. I. 2006, ApJ, 638, 369

Krumholz, M. R., Cunningham, A. J., Klein, R. I., \& McKee, C. F. 2010, ApJ, 713, 1120

Larson, R. B. 1981, MNRAS, 194, 809

Latif, M. A., Schleicher, D. R. G., Spaans, M., \& Zaroubi, S. 2011, MNRAS, 413, L33

Lepp, S., \& Dalgarno, A. 1996, A\&A, 306, L21

Levin, Y. 2007, MNRAS, 374, 515

Li, Y., Klessen, R. S., \& Mac Low, M. 2003, ApJ, 592, 975

Loenen, A. F., Spaans, M., Baan, W. A., \& Meijerink, R. 2008, A\&A, 488, L5

Maloney, P. R., Hollenbach, D. J., \& Tielens, A. G. G. M. 1996, ApJ, 466, 561

Martín, S., Krips, M., Martín-Pintado, J., et al. 2011, A\&A, 527, A36

Matsushita, S., Iono, D., Petitpas, G. R., et al. 2009, ApJ, 693, 56

Meijerink, R., \& Spaans, M. 2005, A\&A, 436, 397

Meijerink, R., Spaans, M., \& Israel, F. P. 2005, in IAU Symp., 235, 58 
Meijerink, R., Spaans, M., \& Israel, F. P. 2007, A\&A, 461, 793

Meijerink, R., Spaans, M., Loenen, A. F., \& van der Werf, P. P. 2011, A\&A, 525, A119

Miller, G. E., \& Scalo, J. M. 1979, ApJS, 41, 513

Myers, P. C., \& Gammie, C. F. 1999, ApJ, 522, L141

Myers, A. T., Krumholz, M. R., Klein, R. I., \& McKee, C. F. 2011, ApJ, 735, 49

Nayakshin, S., Cuadra, J., \& Springel, V. 2007, MNRAS, 379, 21

Ostriker, E. C., \& Shetty, R. 2011, ApJ, 731, 41

Papadopoulos, P. P. 2010, ApJ, 720, 226

Papadopoulos, P. P., Thi, W.-F., Miniati, F., \& Viti, S. 2011, MNRAS, 414, 1705

Parra, R., Conway, J. E., Diamond, P. J., et al. 2007, ApJ, 659, 314

Paumard, T., Genzel, R., Martins, F., et al. 2006, ApJ, 643, 1011

Pérez-Beaupuits, J. P., Aalto, S., \& Gerebro, H. 2007, A\&A, 476, 177

Pérez-Beaupuits, J. P., Spaans, M., van der Tak, F. F. S., et al. 2009, A\&A, 503, 459

Pérez-Beaupuits, J. P., Wada, K., \& Spaans, M. 2011, ApJ, 730, 48

Peters, T., Klessen, R. S., Mac Low, M., \& Banerjee, R. 2010, ApJ, 725, 134

Poelman, D. R., \& Spaans, M. 2006, A\&A, 453, 615

Ruffert, M., \& Arnett, D. 1994, ApJ, 427, 351
Salpeter, E. E. 1955, ApJ, 121, 161

Schleicher, D. R. G., Spaans, M., \& Klessen, R. S. 2010, A\&A, 513, A7

Scoville, N. 2004, in The Neutral ISM in Starburst Galaxies, ed. S. Aalto,

S. Huttemeister, \& A. Pedlar, ASP Conf. Ser., 320, 253

Smail, I., Ivison, R. J., \& Blain, A. W. 1997, ApJ, 490, L5

Spaans, M., \& Meijerink, R. 2008, ApJ, 678, L5

Spaans, M., \& Silk, J. 2000, ApJ, 538, 115

Spoon, H. W. W., Marshall, J. A., Houck, J. R., et al. 2007, ApJ, 654, L49

Stamatellos, D., Whitworth, A. P., \& Hubber, D. A. 2011, ApJ, 730, 32

Truelove, J. K., Klein, R. I., McKee, C. F., et al. 1997, ApJ, 489, L179

van der Werf, P. P., Isaak, K. G., Meijerink, R., et al. 2010, A\&A, 518, L42

van Dokkum, P. G. 2008, ApJ, 674, 29

van Dokkum, P. G., \& Conroy, C. 2010, Nature, 468, 940

van Dokkum, P. G., \& Conroy, C. 2011, ApJ, 735, L13

Wada, K. 2008, ApJ, 675, 188

Wada, K., Papadopoulos, P. P., \& Spaans, M. 2009, ApJ, 702, 63

Wilkins, S. M., Hopkins, A. M., Trentham, N., \& Tojeiro, R. 2008, MNRAS, 391,363

Pages 14 to 25 are available in the electronic edition of the journal at http://www . aanda. org 

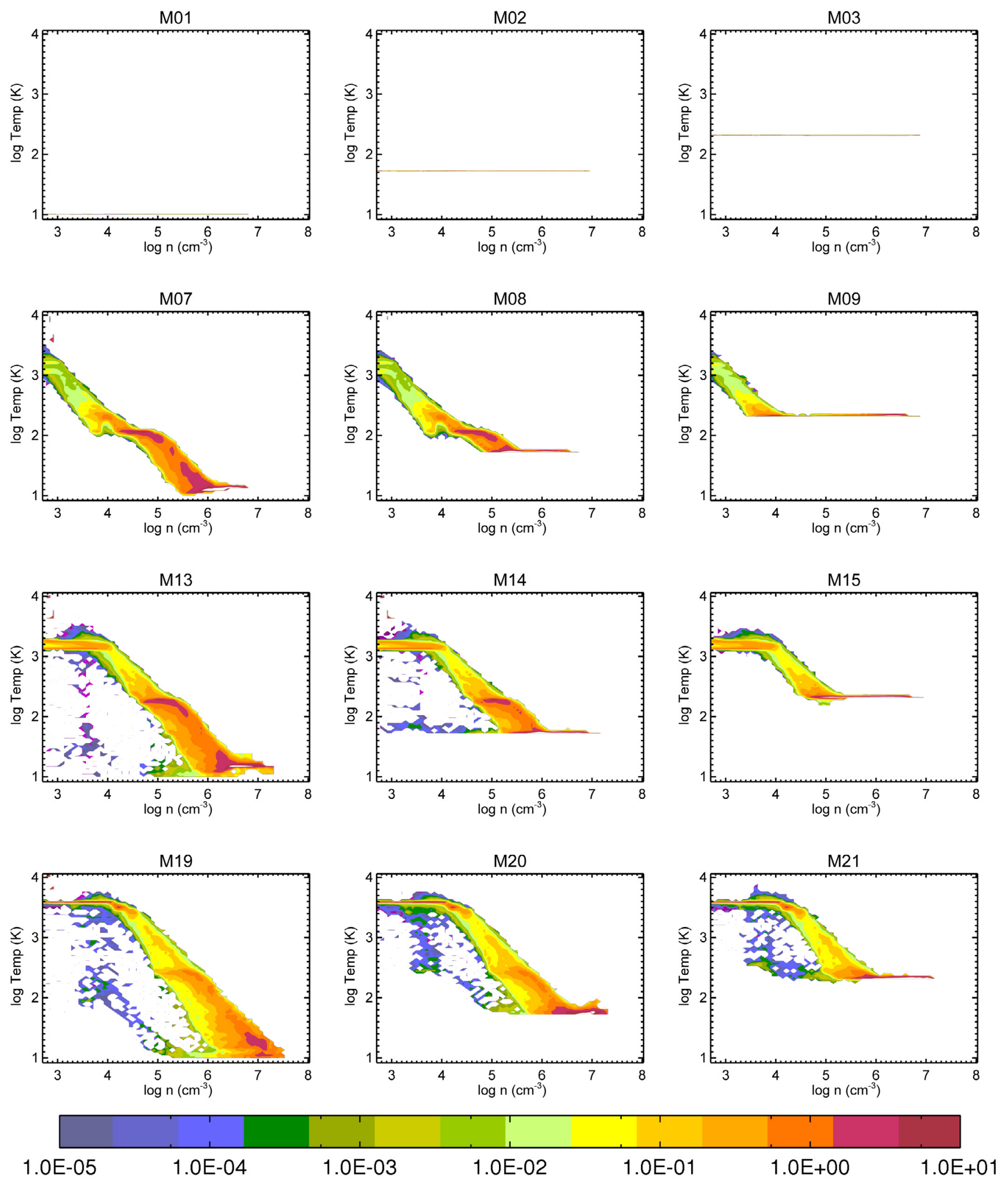

Fig. 6. Phase diagrams for the models with $M_{\mathrm{bh}}=10^{7} M_{\odot}$ and UV $=0$. Temperature is plotted against number density at $t=t_{\mathrm{ff}}=10^{5}$ yr. The diagrams are gridded into $75^{2}$ cells with the weighted masses of the points depicted in color. Red represents a mass of $10 M_{\odot}$ or above and blue is for $10^{-5} M_{\odot}$. Isothermal conditions yield a flat profile. From left to right, the cosmic ray rate increases from 1, 100 to $3000 \times$ Galactic. From top to bottom, the X-ray flux increases from $0,5.1,28$ to $160 \mathrm{erg} \mathrm{s}^{-1} \mathrm{~cm}^{-2}$. 
S. Hocuk and M. Spaans: Star formation in AGN
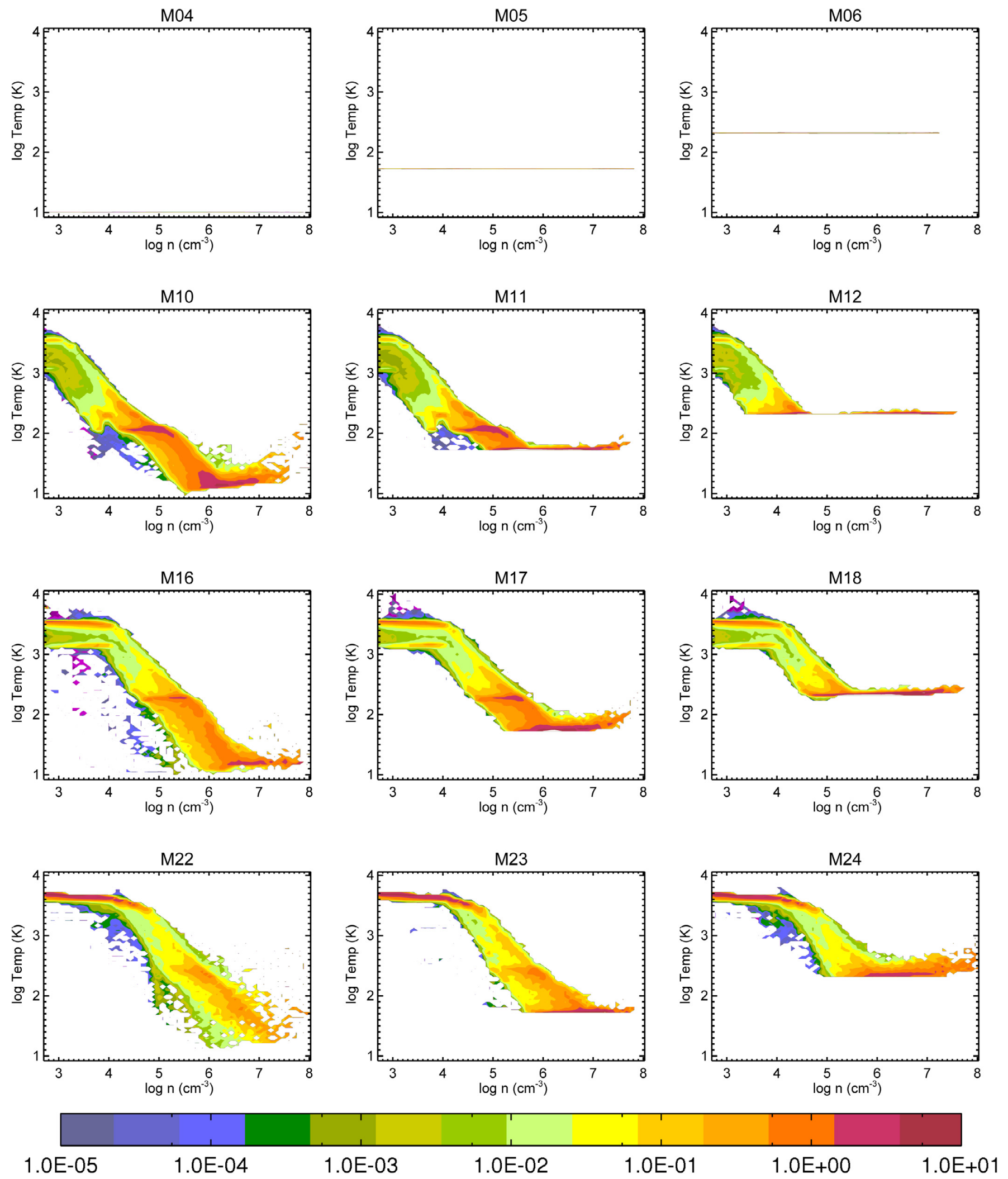

Fig. 7. Phase diagrams for the models with $M_{\mathrm{bh}}=10^{6} M_{\odot}$ and UV $=0$. Temperature is plotted against number density at $t=t_{\mathrm{ff}}=10^{5}$ yr. The diagrams are gridded into $75^{2}$ cells with the weighted masses of the points depicted in color. Red represents a mass of $10 M_{\odot}$ or above and blue is for $10^{-5} M_{\odot}$. Isothermal conditions yield a flat profile. From left to right, the cosmic ray rate increases from 1, 100 to $3000 \times$ Galactic. From top to bottom, the X-ray flux increases from $0,5.1,28$ to $160 \mathrm{erg} \mathrm{s}^{-1} \mathrm{~cm}^{-2}$. 

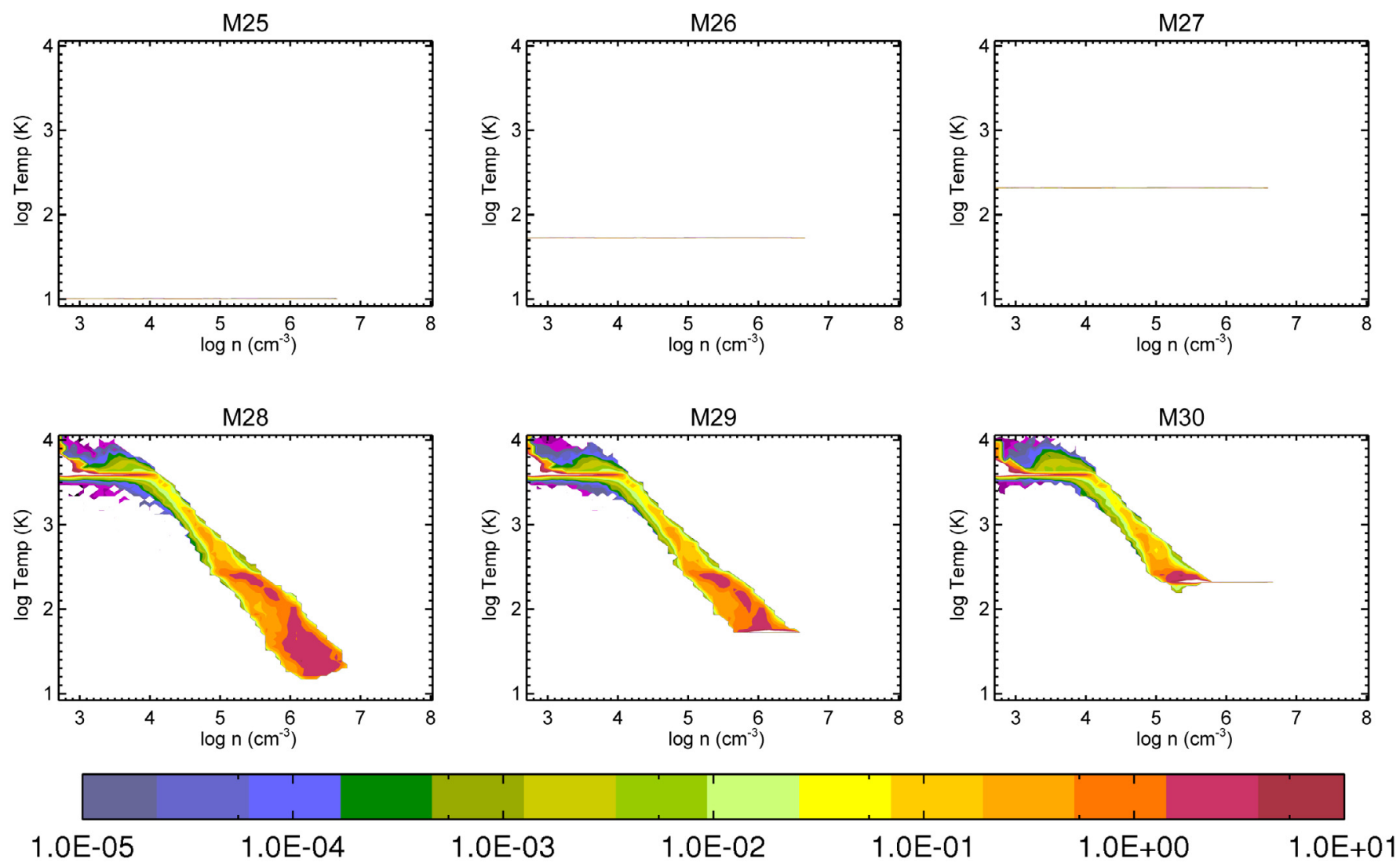

Fig. 8. Phase diagrams for the models with $M_{\mathrm{bh}}=10^{8} M_{\odot}$ and UV=0. Temperature is plotted against number density at $t=t_{\mathrm{ff}}=10^{5}$ yr. The diagrams are gridded into $75^{2}$ cells with the weighted masses of the points depicted in color. Red represents a mass of $10 M_{\odot}$ or above and blue is for $10^{-5} M_{\odot}$. Isothermal conditions yield a flat profile. From left to right, the cosmic ray rate increases from 1, 100 to $3000 \times$ Galactic. From top to bottom, the X-ray flux increases from 0 to $160 \mathrm{erg} \mathrm{s}^{-1} \mathrm{~cm}^{-2}$. 

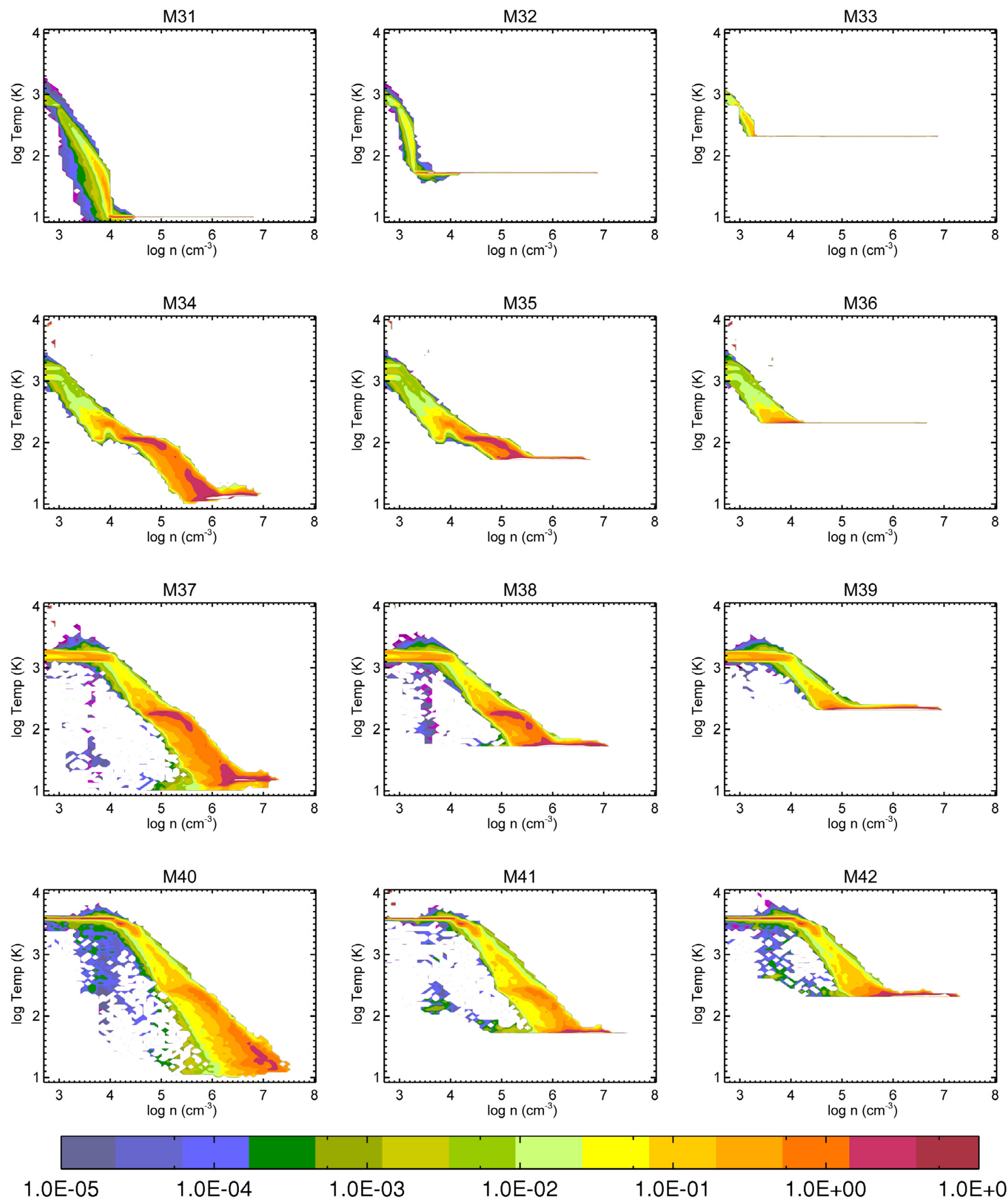

$1.0 \mathrm{E}-03$

1.0E-02

1.0E-01

$1.0 \mathrm{E}+00$

$1.0 \mathrm{E}+01$

Fig. 9. Phase diagrams for the models including UV. The UV flux used in these models is $10^{2.5} \mathrm{G}_{0}$. Similar to the figures above, Figs. 6 to 8 , the images display the temperature against number density at $t=t_{\mathrm{ff}}=10^{5} \mathrm{yr}$. The diagrams are gridded into $75^{2}$ cells with the weighted masses of the points depicted in color. Red represents a mass of $10 M_{\odot}$ or above and blue is for $10^{-5} M_{\odot}$. From left to right, the cosmic ray rate increases from 1 , 100 to $3000 \times$ Galactic. From top to bottom, the X-ray flux increases from 0 to $160 \mathrm{erg} \mathrm{s}^{-1} \mathrm{~cm}^{-2}$. These UV models are simulated for a black hole mass of $M_{\mathrm{bh}}=10^{7} M_{\odot}$. 

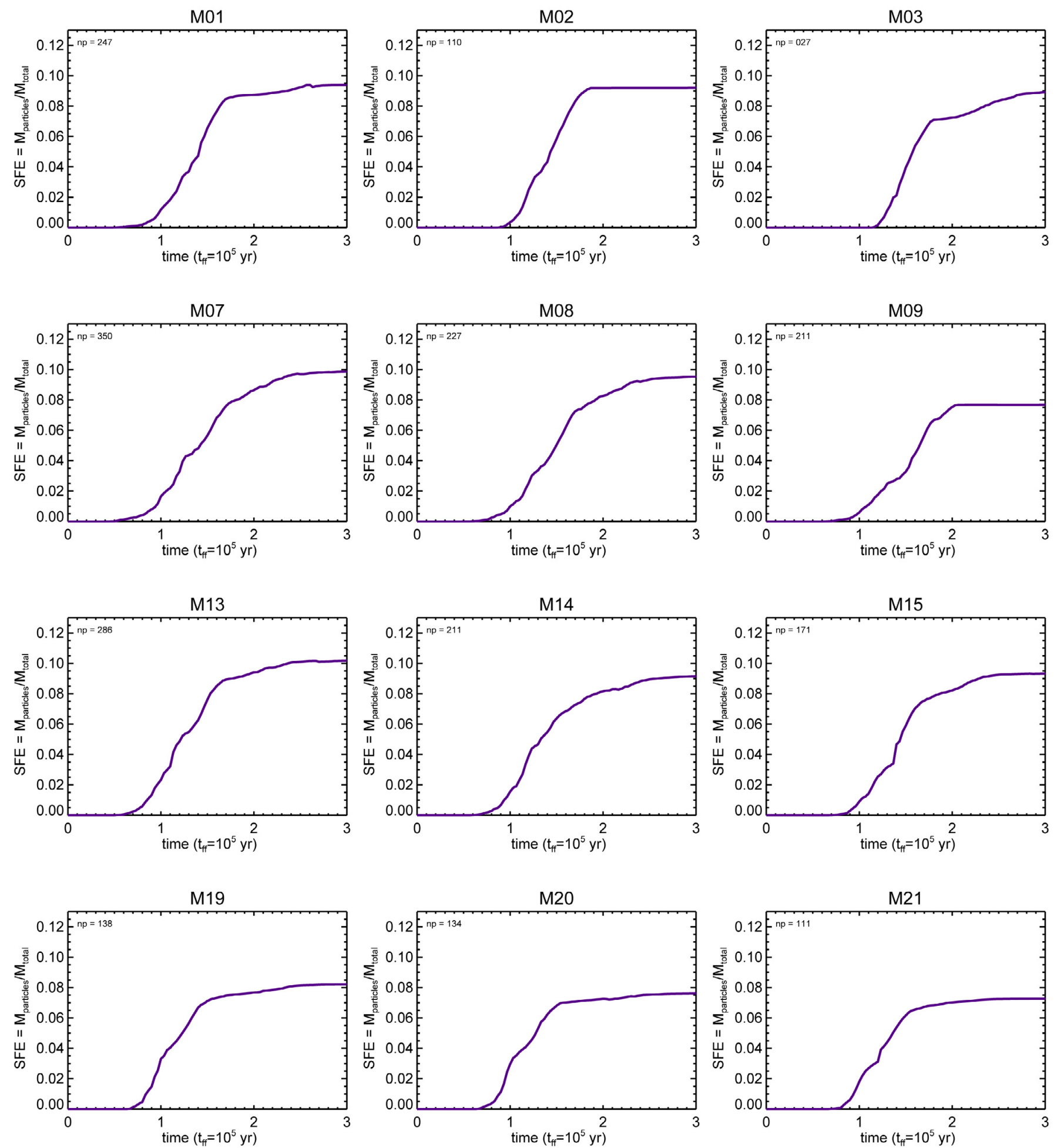

Fig. 10. SFEs for the models with $M_{\mathrm{bh}}=10^{7} M_{\odot}$ and $\mathrm{UV}=0$. The ratio of the total sink particle mass over the total initial gas mass is plotted against time (in free-fall units). From left to right, the cosmic ray rate increases from 1,100 to $3000 \times$ Galactic. From top to bottom, the X-ray flux increases from $0,5.1,28$ to $160 \mathrm{erg} \mathrm{s}^{-1} \mathrm{~cm}^{-2}$. The total number of sink particles formed during the run is given in the upper left corner of each panel. 

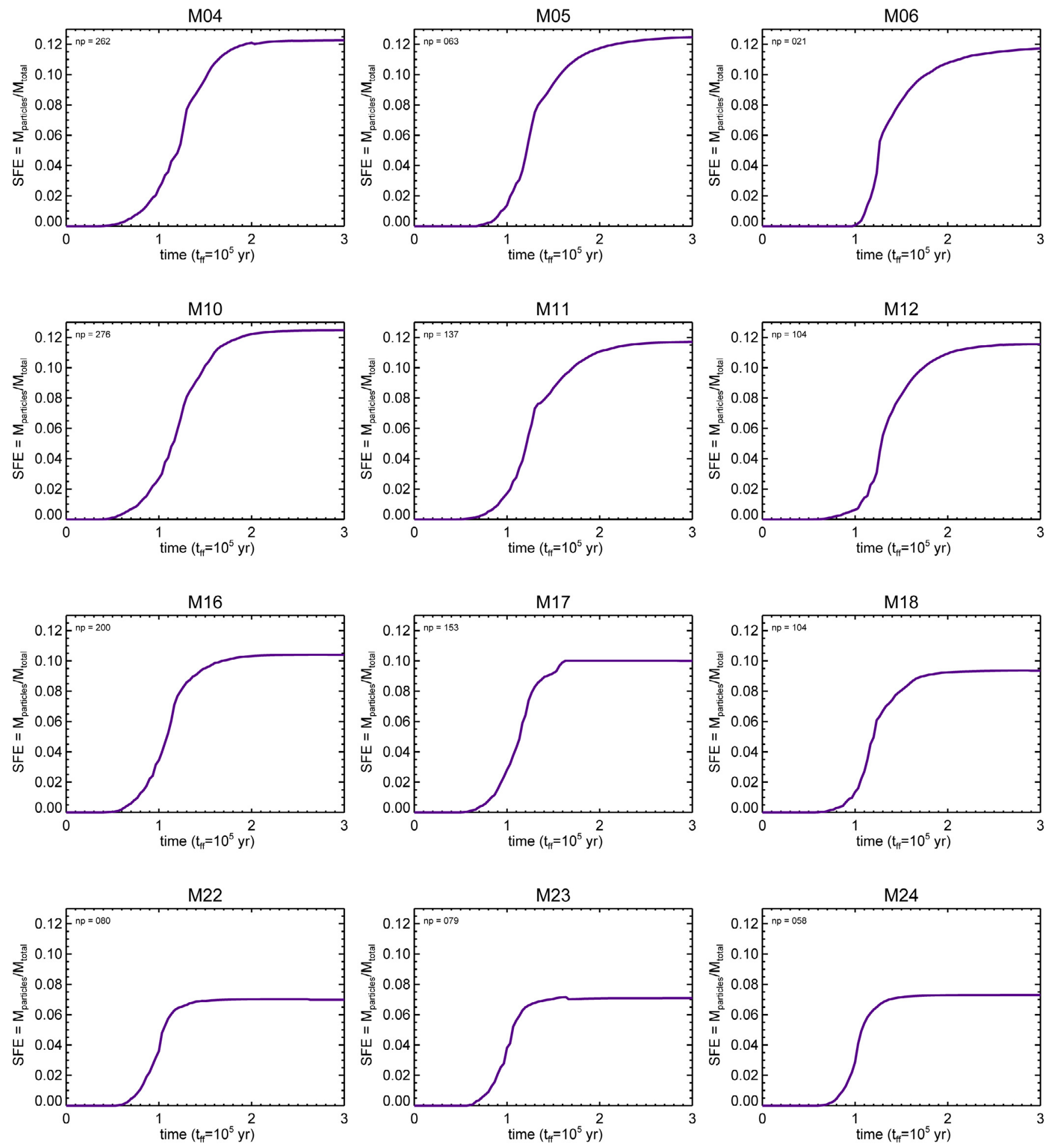

Fig. 11. SFEs for the models with $M_{\mathrm{bh}}=10^{6} M_{\odot}$ and $\mathrm{UV}=0$. The ratio of the total sink particle mass over the total initial gas mass is plotted against time (in free-fall units). From left to right, the cosmic ray rate increases from 1, 100 to $3000 \times$ Galactic. From top to bottom, the X-ray flux increases from $0,5.1,28$ to $160 \mathrm{erg} \mathrm{s}^{-1} \mathrm{~cm}^{-2}$. The total number of sink particles formed during the run is given in the upper left corner of each panel. 

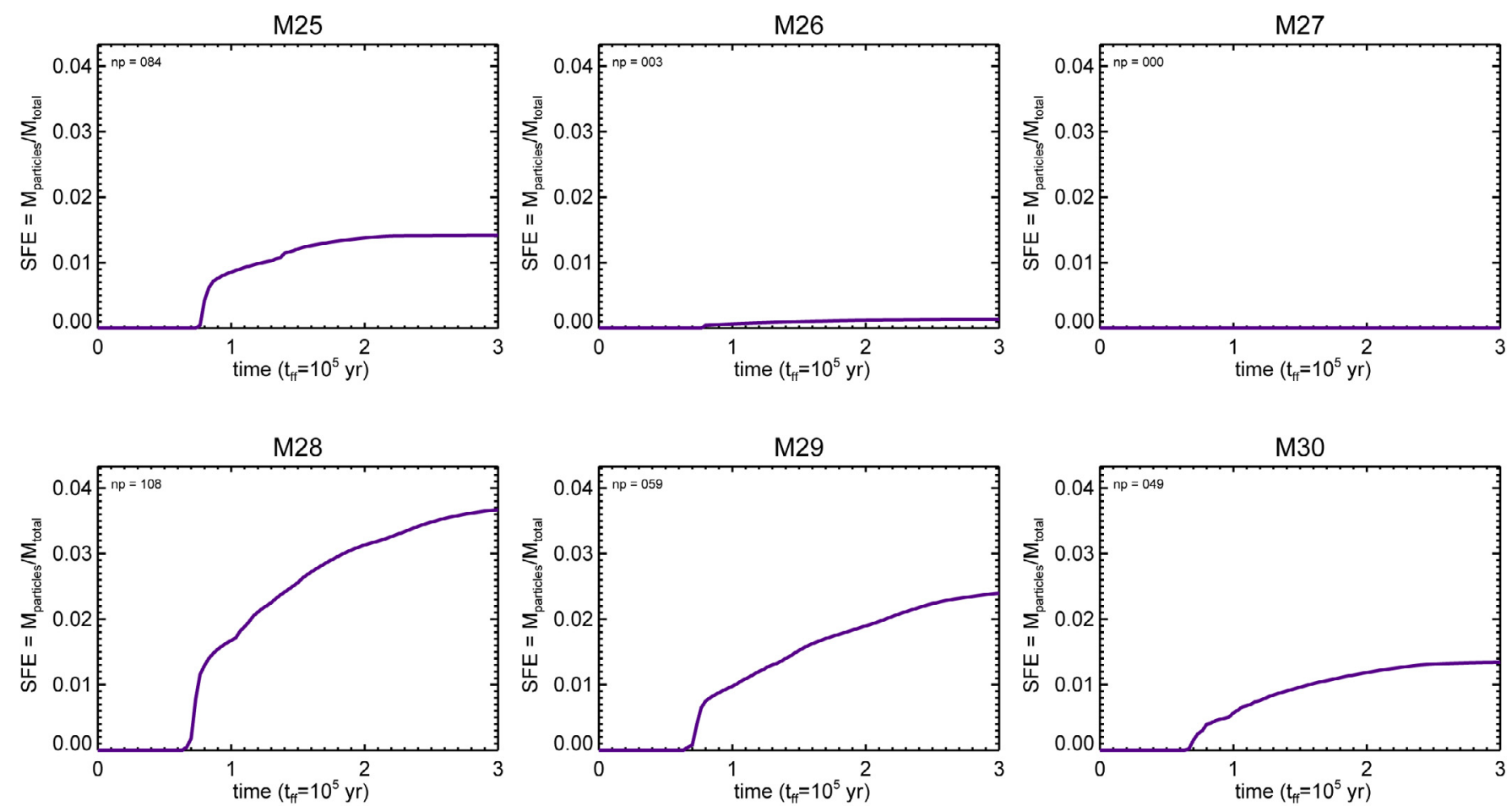

Fig. 12. SFEs for the models with $M_{\mathrm{bh}}=10^{8} M_{\odot}$ and $\mathrm{UV}=0$. The ratio of the total sink particle mass over the total initial gas mass is plotted against time (in free-fall units). Note that the $y$-axis range in this figure differs from the other (SFE) figures. From left to right, the cosmic ray rate increases from 1, 100 to $3000 \times$ Galactic. From top to bottom, the X-ray flux increases from 0 to $160 \mathrm{erg} \mathrm{s}^{-1} \mathrm{~cm}^{-2}$. The total number of sink particles formed during the run is given in the upper left corner of each panel. 

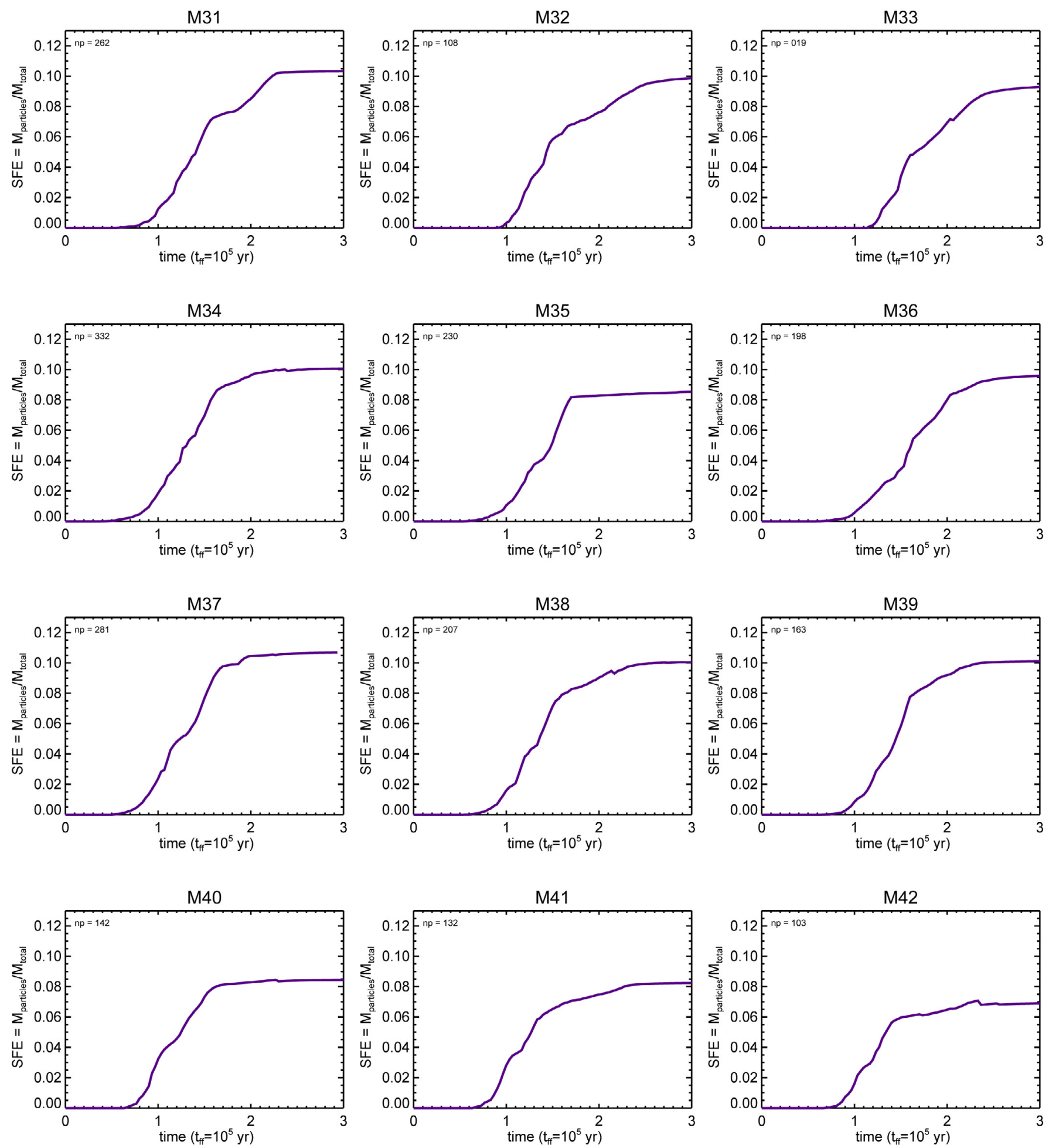

Fig. 13. SFEs for the models with UV. The UV flux used in these models is $10^{2.5} \mathrm{G}_{0}$. Similar to the figures above, Figs. 10 to 12 , the images show the ratio of the total sink particle mass over the total initial gas mass and is plotted against time (in free-fall units). From left to right, the cosmic ray rate increases from 1, 100 to $3000 \times$ Galactic. From top to bottom, the X-ray flux increases from 0,5.1, 28 to $160 \mathrm{erg} \mathrm{s}^{-1} \mathrm{~cm}^{-2}$. These UV models are simulated for a a black hole mass of $M_{\mathrm{bh}}=10^{7} M_{\odot}$. The total number of sink particles formed during the run is given in the upper left corner of each panel. 

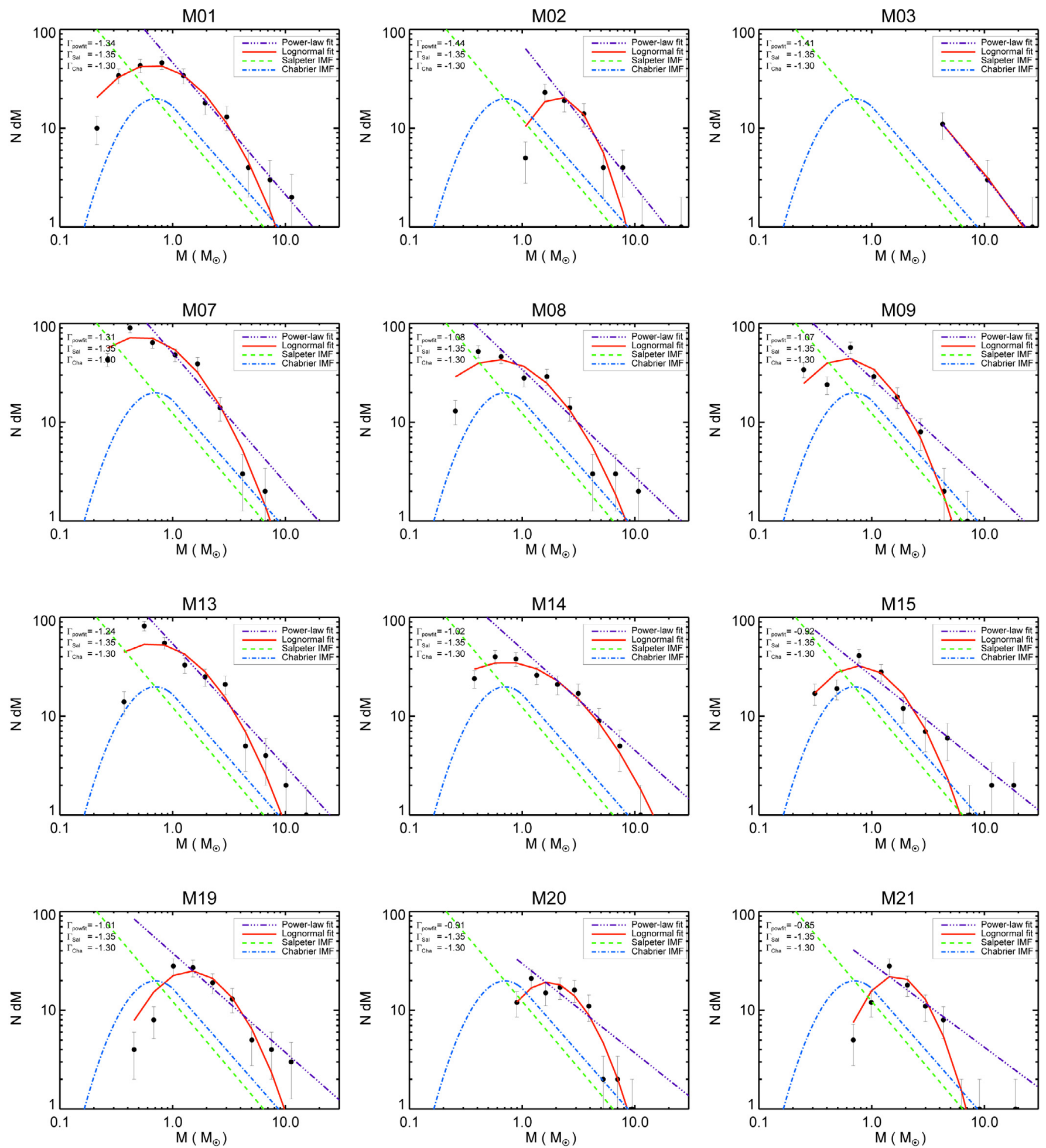

Fig. 14. IMFs for the models with $M_{\mathrm{bh}}=10^{7} M_{\odot}$ and UV $=0$. The images display the time-averaged IMFs between 1 and 3 free-fall times, where $t_{\mathrm{ff}}=10^{5} \mathrm{yr}$. From left to right, the cosmic ray rate increases from 1, 100 to $3000 \times$ Galactic. From top to bottom, the X-ray flux increases from 0 , 5.1, 28 to $160 \mathrm{erg} \mathrm{s}^{-1} \mathrm{~cm}^{-2}$. In each image, for comparison purposes, the Salpeter IMF (green dashed) and the Chabrier IMF (blue dot-dashed) are displayed as fitted to our fiducial model. Two best fits are applied to the data, a linear fit and a lognormal fit, and are shown as purple and red lines. With the exception of the lognormal fit, the slopes above the turn-over mass are given in the upper left corner. 
S. Hocuk and M. Spaans: Star formation in AGN
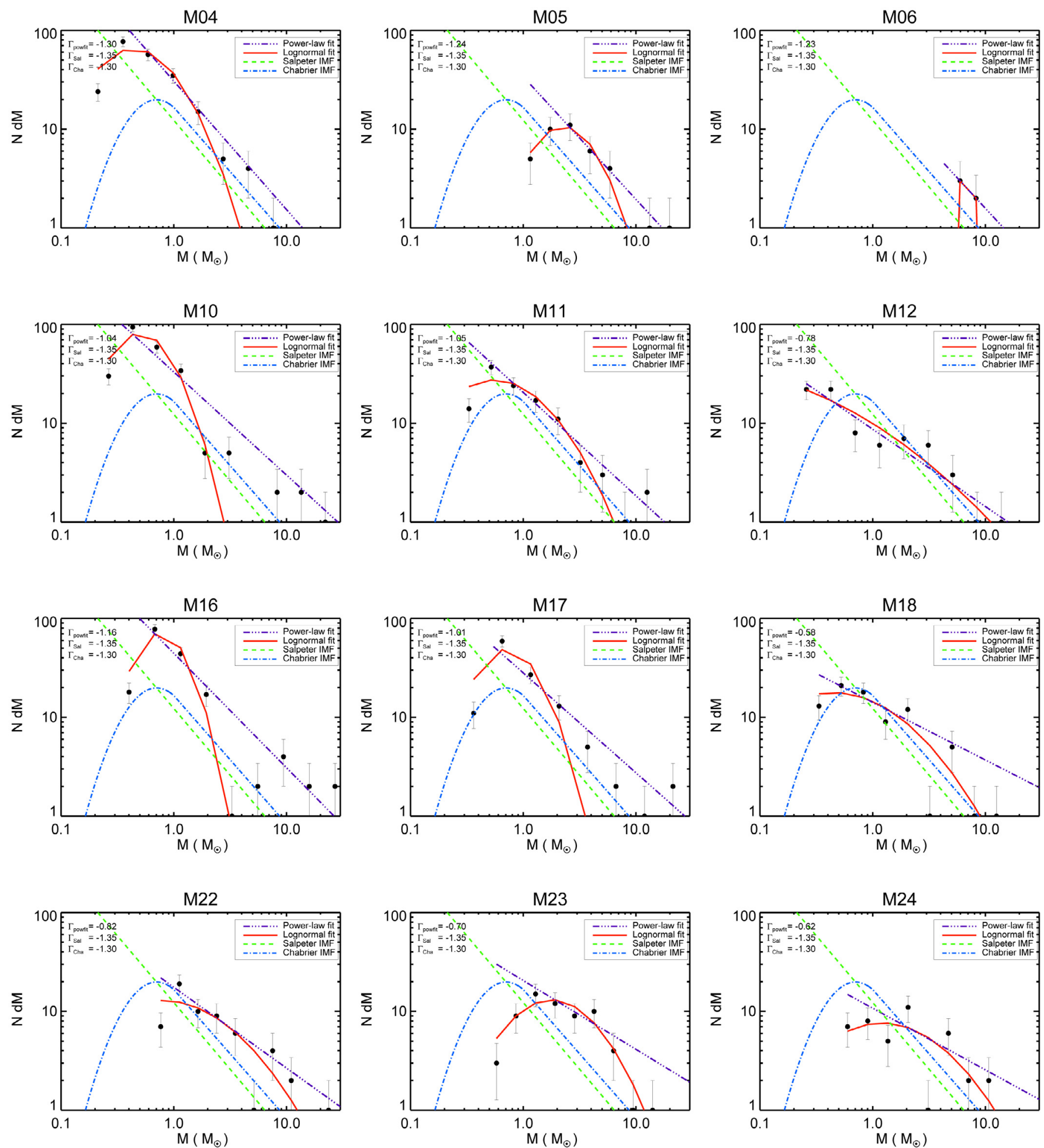

Fig. 15. IMFs for the models with $M_{\mathrm{bh}}=10^{6} M_{\odot}$ and UV $=0$. The images display the time-averaged IMFs between 1 and 3 free-fall times, where $t_{\mathrm{ff}}=10^{5} \mathrm{yr}$. From left to right, the cosmic ray rate increases from 1, 100 to $3000 \times$ Galactic. From top to bottom, the X-ray flux increases from 0 , 5.1, 28 to $160 \mathrm{erg} \mathrm{s}^{-1} \mathrm{~cm}^{-2}$. In each image, for comparison purposes, the Salpeter IMF (green dashed) and the Chabrier IMF (blue dot-dashed) are displayed as fitted to our fiducial model. Two best fits are applied to the data, a linear fit and a lognormal fit, and are shown as purple and red lines. With the exception of the lognormal fit, the slopes above the turn-over mass are given in the upper left corner. 

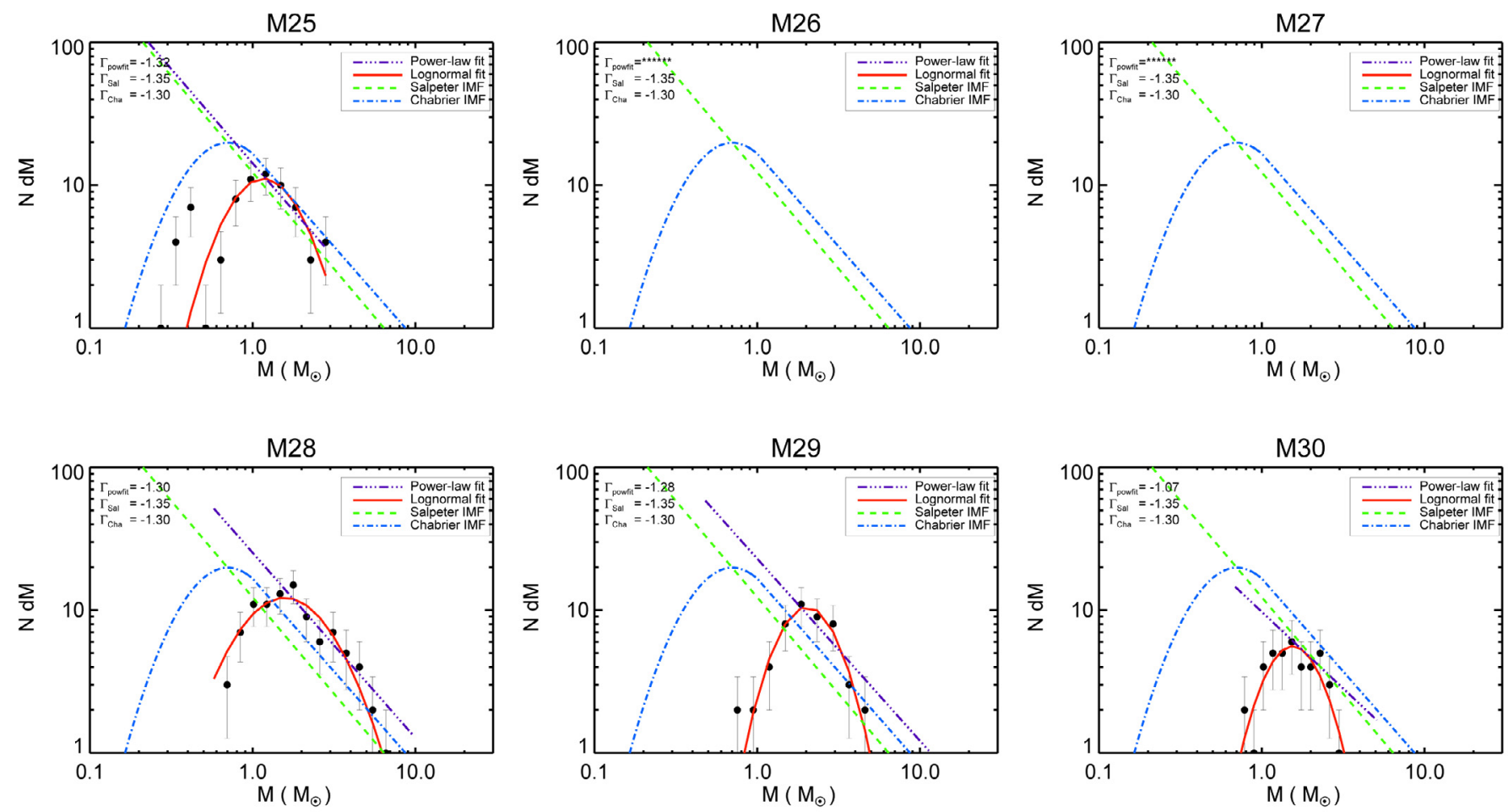

Fig. 16. IMFs for the models with $M_{\mathrm{bh}}=10^{8} M_{\odot}$ and $\mathrm{UV}=0$. The images display the time-averaged IMFs between 1 and 3 free-fall times, where $t_{\mathrm{ff}}=10^{5} \mathrm{yr}$. From left to right, the cosmic ray rate increases from 1, 100 to $3000 \times$ Galactic. From top to bottom, the X-ray flux increases from 0 to $160 \mathrm{erg} \mathrm{s}^{-1} \mathrm{~cm}^{-2}$. In each image, for comparison purposes, the Salpeter IMF (green dashed) and the Chabrier IMF (blue dot-dashed) are displayed as fitted to our fiducial model. Two best fits are applied to the data, a linear fit and a lognormal fit, and are shown as purple and red lines. With the exception of the lognormal fit, the slopes above the turn-over mass are given in the upper left corner. 

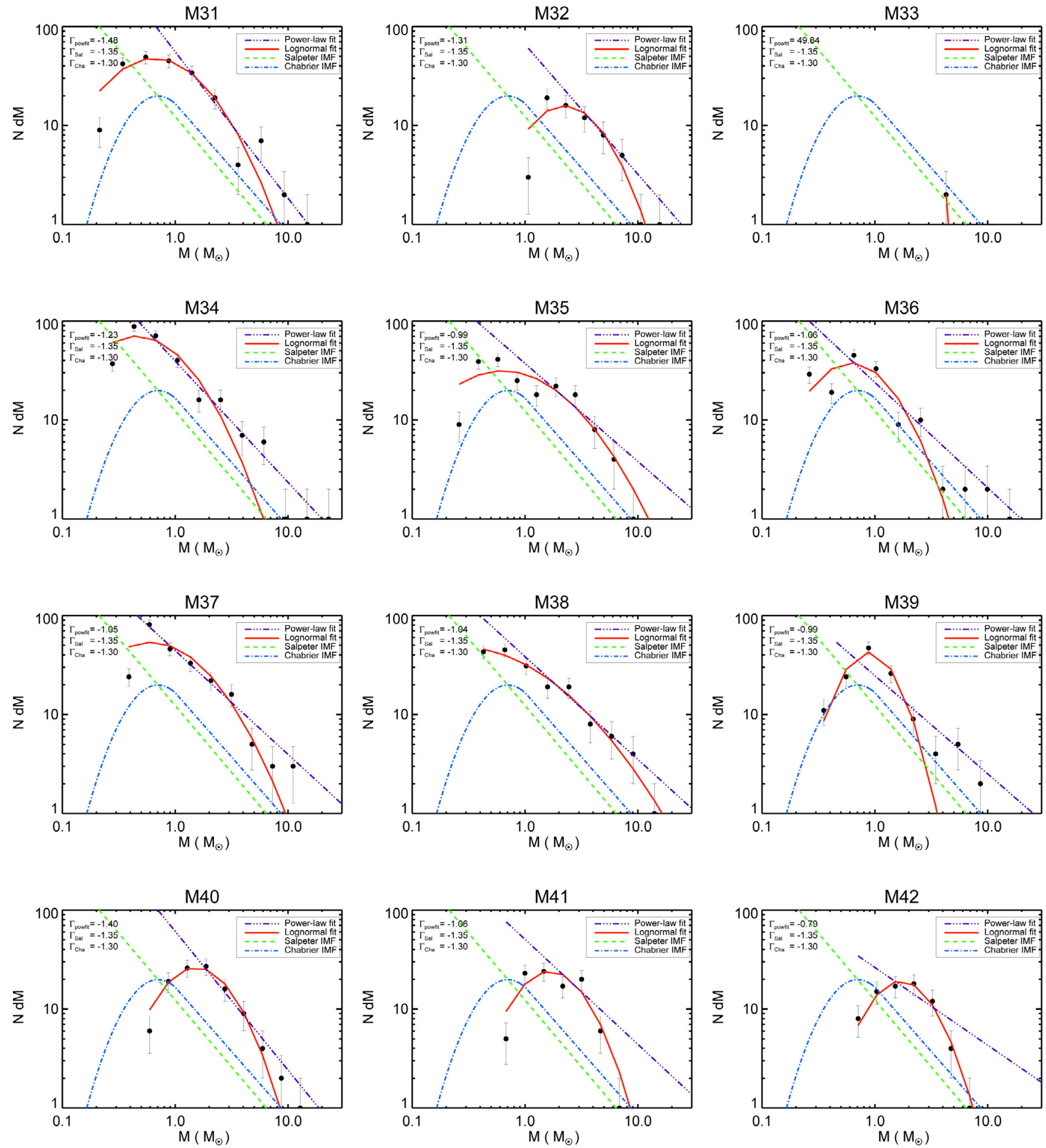

Fig. 17. IMFs for the models with UV. The UV flux used in these models is $10^{2.5} \mathrm{G}_{0}$. Similar to the figures above, Figs. 14 to 16 , the images display the time-averaged IMFs between 1 and 3 free-fall times, where $t_{\mathrm{ff}}=10^{5} \mathrm{yr}$. From left to right, the cosmic ray rate increases from 1, 100 to 3000 $\times$ Galactic. From top to bottom, the X-ray flux increases from 0, 5.1, 28 to $160 \mathrm{erg} \mathrm{s}^{-1} \mathrm{~cm}^{-2}$. In each image, for comparison purposes, the Salpeter IMF (green dashed) and the Chabrier IMF (blue dot-dashed) are displayed as fitted to our fiducial model. Two best fits are applied to the data, a linear fit and a lognormal fit, and are shown as purple and red lines. With the exception of the lognormal fit, the slopes above the turn-over mass are given in the upper left corner. These UV models are simulated for a a black hole mass of $M_{\mathrm{bh}}=10^{7} M_{\odot}$. 